\title{
Comprehensive analysis of differentially expressed genes and transcriptional regulation induced by salt stress in two contrasting cotton genotypes
}

Zhen Peng ${ }^{1,2}$, Shoupu He ${ }^{1}$, Wenfang Gong ${ }^{1}$, Junling Sun ${ }^{1}$, Zhaoe Pan ${ }^{1}$, Feifei Xu' , Yanli Lu ${ }^{2 *}$ and Xiongming Du ${ }^{1 *}$

\begin{abstract}
Background: Cotton (Gossypium spp.) is one of the major fibre crops of the world. Although it is classified as salt tolerant crop, cotton growth and productivity are adversely affected by high salinity, especially at germination and seedling stages. Identification of genes and miRNAs responsible for salt tolerance in upland cotton (Gossypium hirsutum L.) would help reveal the molecular mechanisms of salt tolerance. We performed physiological experiments and transcriptome sequencing (mRNA-seq and small RNA-seq) of cotton leaves under salt stress using Illumina sequencing technology.

Results: We investigated two distinct salt stress phases_-dehydration (4 h) and ionic stress (osmotic restoration; $24 \mathrm{~h}$ ) that were identified by physiological changes of 14-day-old seedlings of two cotton genotypes, one salt tolerant and the other salt sensitive, during a 72-h NaCl exposure. A comparative transcriptomics was used to monitor gene and miRNA differential expression at two time points ( 4 and $24 \mathrm{~h}$ ) in leaves of the two cotton genotypes under salinity conditions. The expression patterns of differentially co-expressed unigenes were divided into six groups using short time-servies expression miner software. During a 24-h salt exposure, 819 transcription factor unigenes were differentially expressed in both genotypes, with 129 unigenes specifically expressed in the salt-tolerant genotype. Under salt stress, 108 conserved miRNAs from known families were differentially expressed at two time points in the salt-tolerant genotype. We further analyzed the predicted target genes of these miRNAs along with the transcriptome for each time point. Important expressed genes encoding membrane receptors, transporters, and pathways involved in biosynthesis and signal transduction of calcium-dependent protein kinase, mitogen-activated protein kinase, and hormones (abscisic acid and ethylene) were up-regulated. We also analyzed the salt stress response of some key miRNAs and their target genes and found that the expressions of five of nine target genes exhibited significant inverse correlations with their corresponding miRNAs. On the basis of these results, we constructed molecular regulatory pathways and a potential regulatory network for these salt-responsive miRNAs.
\end{abstract}

Conclusions: Our comprehensive transcriptome analysis has provided new insights into salt-stress response of upland cotton. The results should contribute to the development of genetically modified cotton with salt tolerance.

Keywords: Cotton, Salt stress, Leaf transcriptome, Transcription factor, MicroRNA

\footnotetext{
* Correspondence: dujeffrey8848@hotmail.com; yanli.lu82@hotmail.com

${ }^{1}$ State Key Laboratory of Cotton Biology/Institute of Cotton Research,

Chinese Academy of Agricultural Sciences, 455000 Anyang, Henan, China

${ }^{2}$ Maize Research Institute, Sichuan Agricultural University, 611130 Wenjiang,

Sichuan, China
}

\section{Biomed Central




\section{Background}

High soil salinity, a devastating environmental stress, typically causes major reductions in crop productivity and quality [1]. Over 800 million hectares, equivalent to $6.5 \%$ of the world's total land area, are currently estimated to be impacted by high salt concentration [2]. In China, approximately 100 million hectares distributed over 16 provinces have been affected by increased salinity [3]. To stabilize global crop production, the problem of salinity must be urgently addressed.

Salt stress generally induces a combination of dehydration/osmotic-related effects and damage as a consequence of excess sodium ions that greatly affect plant growth and crop production [4]. Osmotic stress and $\mathrm{Na}^{+}$stress are considered to be the two major components of the plant salt-stress response [5]. Plants employ various mechanisms to deal with salt stress; these mechanisms include minimization of the amount of salt taken up by roots and its partitioning at tissue and cellular levels to avoid buildup of toxic concentrations in the cytosol of functional leaves $[6,7]$. Much effort has been devoted to revealing the molecular mechanisms of plant salt tolerance, with the ultimate goal of improving salt tolerance of crop plants.

Cotton (Gossypium hirsutum L.) is not only the world's leading textile fiber, but is also a major oil crop. Although cotton is the second most salt-tolerant herbaceous crop $[8,9]$, its growth and productivity are adversely affected by high salinity, especially at germination and the young seedling stage [10]. Salinity suppresses primary root growth [11], inhibits the length and numbers of secondary roots [12], and limits photosynthesis and respiration, flowering, boll and fiber quality, and ion uptake in cotton, resulting in significant yield losses [13]. Salt stress has also been found to regulate the expression level of many genes in different bio-processes and pathways, including morphological adaptation, maintenance of ion homeostasis, cell signal transduction, and oxidative stress mitigation [14-16]. Identifying salt-tolerance genes is an important component of the breeding of salt-tolerant crop plants through genetic engineering. Although many genes controlling response to high salinity have been identified in model plants, only a few salt stress-inducible genes, such as NHX1 (GhNHX1) [17], DREB (GhDREB1) [18], ERF (GhERF2- GhERF6) [19-21], NAC (GhNAC1-GhNAC6) [22], metallothionein (GhMT3a) [23], GhMPK2 [24], GhMKK1 [25], and CCCH-type zinc finger (GhZFP1) [26] have been documented in cotton. With recent advances in genomic sequencing and transcriptome mapping (microarray and high-throughput sequencing), some salt-related genes and regulatory factors have been identified in cotton on a large scale at the genome-wide level [27-33]. Nevertheless, the molecular basis of cotton tolerance to salt stress remains to be discovered.
Small RNAs are important post-transcriptional regulators of gene expression. miRNAs are a highly conserved class of endogenous, non-coding RNAs that range in length from 19 to 25 nucleotides (nt) [34]. In plants, cleavage or translational repression of target mRNA appears to be the prevalent method of posttranscriptional regulation $[35,36]$. Current findings that some plant miRNAs respond to stress conditions and that some miRNA targets are stress-related genes suggest that miRNAs play important roles in plant stress response. miRNA expression profiles in response to salt stress have been analyzed in Arabidopsis thaliana [37], Oryza sativa [38], Zea mays [39], Gossypium hirsutum [40], and Caragana intermedia [41]. In addition to studying the effects of stress on miRNAs, the identification of miRNA targets is important. Among cotton cultivars, however, some miRNAs related to salt tolerance have been found to differ [42-45]. Little is known regarding their expression profiles in response to salt stress, and their roles in salt adaptation remain unclear.

Transcriptome (mRNA-seq and small RNA-seq) analysis has recently emerged as a powerful approach to excavate large numbers of genes and miRNA sequences $[45,46]$. Although some differentially expressed salt-related genes have been identified by this technique after shortor long-term salt treatment, comparative transcriptional changes in response to salt stress between early and late periods have not been reported. An additional limitation is that most reported gene expression profiles related to salt resistance have been derived from a single genotype [47-49]. In those studies, it was consequently difficult to accurately distinguish true salt tolerance-related genes from salt-responsive ones. Furthermore, most research for salt stress focused on root $[31,46]$. However, the previous results show that when subjected to salt stress in upland cotton, mostly absorbed $\mathrm{Na}^{+}$accumulated in the shoot through the roots, and the root of $\mathrm{K}^{+}$transport to the leaves to maintain high $\mathrm{K}^{+} / \mathrm{Na}^{+}$in the leaves [9]. Otherwise, in our study, $\mathrm{Na}^{+}$concentration in leaf also showed significant difference between two lines (Figure 1E), lower $\mathrm{Na}^{+}$concentration could reduce the damage of leaf photosynthesis, further improved the salt tolerance. These results indicated that the shoot, especially the leaves of salt tolerant cotton must have some special mechanisms to alleviate $\mathrm{Na}^{+}$toxicity under salt stress, therefore, we chose the leaf as object of study. Thus, comparative analyses of gene and miRNA expression in seedling leaves during two stages of salt stress may further elucidate molecular mechanisms of salt tolerance in cotton.

To obtain insights into the initial transcriptional regulation and differential expression of functional genes in response to high salinity, we exposed two cotton genotypes (salt-tolerant Earlistaple 7 and salt-sensitive Nan Dan Ba 

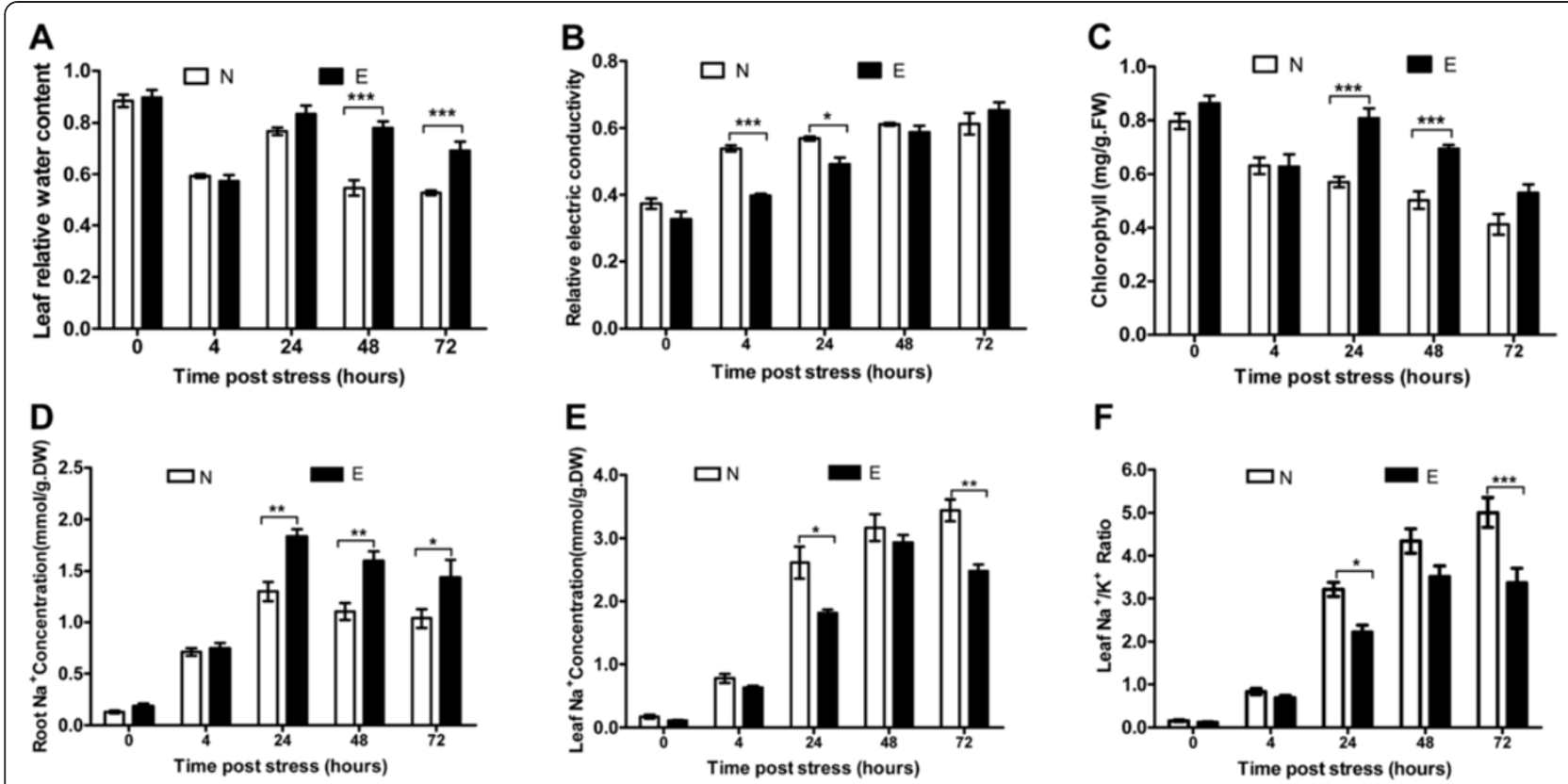

Figure 1 Physiological analysis of Earlistaple 7 and Nan Dan Ba Di Da Hua in response to various durations of salt stress. (A) Leaf relative water content (RWC) levels; (B) Leaf relative electrical conductivity (REC); (C) Leaf chlorophyll-a and chlorophyll-b content; (D) Root Na ${ }^{+}$ concentration; (E) Leaf $\mathrm{Na}^{+}$concentration; (F) Ratio of leaf $\mathrm{K}^{+} / \mathrm{Na}^{+}$concentration in $200 \mathrm{mM} \mathrm{NaCl}$-treated and non-treated samples. Data represent means \pm SE of three independent experiments $(n=3$ or 9$)$; $P<0.05$; ${ }^{* *} P<0.01$; ${ }^{* *} P<0.001$.

Di Da Hua) to salt stress for 0-72 h. We then compared dynamic changes in ion accumulation and osmotic adjustment between Earlistaple 7 and Nan Dan Ba Di Da Hua and analyzed the expression profiles of responsive genes and miRNAs. According to their expression patterns, salt stress-inducible genes could be broadly discerned as early-, late-, and sustained-induced regulated genes, and their functional significance was characterized. We also identified and characterized conserved miRNAs in the two genotypes at two time points ( 4 and $24 \mathrm{~h}$ ) under salt stress. Putative target genes of these miRNAs were predicted from the transcripts by computational methods. We established a model of the miRNA-mediated regulatory network by combining the mRNA-seq and small RNA-seq information. Our comprehensive physiological- and molecular-level analysis of salt stress responses should facilitate illumination of the mechanisms of salt stress tolerance in G. hirsutum.

\section{Results}

Physiological differences between salt-sensitive and salt-tolerant genotypes under salinity stress

Genotypes Nan Dan Ba Di Da Hua and Earlistaple 7 were subjected to short-term salinity stress $(200 \mathrm{mM}$ $\mathrm{NaCl}$ ) at the seedling stage for $4,24,48$, and 72-h. Seedling response to salt was evaluated based on leaf relative water content (RWC), relative electrical conductivity (REC), and chlorophyll and ion $\left(\mathrm{Na}^{+}\right.$and $\left.\mathrm{K}^{+}\right)$content of leaves and roots (Figure 1).
Highly significant $(P<0.001)$ differences were observed in leaf RWC and chlorophyll content between the two genotypes after 48- and 24-h salt treatments, respectively, although these values were sharply decreased in both genotypes after $4 \mathrm{~h}$ of exposure to $\mathrm{NaCl}$ compared with controls (Figure 1A, C). After 4 and $24 \mathrm{~h}$ of treatment, the REC of Nan Dan Ba Di Da Hua was highly significantly $(P<0.001)$ increased throughout the duration of stress, while that of Earlistaple 7 was maintained at a lower level. After $48 \mathrm{~h}$, however, the RECs of the two genotypes were similar (Figure 1B). As shown in Figure 1D-F, leaf and root $\mathrm{Na}^{+}$concentrations increased in both genotypes under salinity stress. Characteristically, Nan Dan Ba Di Da Hua roots accrued less $\mathrm{Na}^{+}$as compared with Earlistaple 7 (Figure 1D), whereas Earlistaple 7 contained a significantly lower level of leaf $\mathrm{Na}^{+}$ (Figure 1E, F). This difference can probably be attributed to the higher $\mathrm{Na}^{+}$absorption capacity of roots of salt-tolerant Earlistaple 7 compared with salt-sensitive Nan Dan Ba Di $\mathrm{Da}$ Hua, which prevents the transport of excessive $\mathrm{Na}^{+}$to the seedling shoots.

Visual damage owing to salinity stress appeared on the leaves of Nan Dan Ba Di Da Hua beginning approximately 24 to $72 \mathrm{~h}$ after $200-\mathrm{mM} \mathrm{NaCl}$ treatment. After $0.5 \mathrm{~h}$, distinct wilting and dehydration caused by osmotic stress [50] was observed on leaves of both genotypes. By $4 \mathrm{~h}$, both genotypes showed more severe wilting, which was followed by gradual recovery to an upright position $(24 \mathrm{~h})$. The restoration of Earlistaple 7 was obviously 
better than that of Nan Dan Ba Di Da Hua, which experienced slight wilting of cotyledons and old leaves, and dry necrosis of leaf edges. After $48 \mathrm{~h}$, these phenomena were markedly more noticeable in both genotypes, although plants of Earlistaple 7 were in much better condition than those of Nan Dan Ba Di Da Hua. According to the above results, significant physiological and morphological differences were observed at different salttreatment time points in response to salt stress, which was accordingly divided into two typical phases: dehydration stress $(4 \mathrm{~h})$ and ionic stress $(24 \mathrm{~h})$. We therefore selected these two time points for further study.

\section{De novo mRNA-seq assembly and annotation of non-redundant unigenes}

Six cDNA and six small RNA libraries were constructed from total RNA extracted from 14-day-old seedling leaves of two upland cottons (cultivars Nan Dan Ba Di Da Hua and Earlistaple 7) treated with $200 \mathrm{mM} \mathrm{NaCl}$ (or water as a control) for 4 and $24 \mathrm{~h}$.

Sample data from the six different libraries are summarized in Table 1. After quality control, approximately $169,850,000$ valid reads and roughly $7.9 \mathrm{~Gb}$ of nucleotides were obtained. The overall data retention rate was high (average of $82.72 \%$ ), and the data quality was acceptable. Because the whole-genome sequence of upland cotton is currently not publically available, the valid reads from the six libraries were merged for de novo assembly (Table 1). After removal of repeats from the spliced sequences, 415,429 transcripts with lengths $\geq 200$ bp were obtained. The total length of all transcripts was approximately $236 \mathrm{Mb}$. The longest transcript for each locus was taken as the unigene, resulting in 143,080 unigenes comprising about $54 \mathrm{Mb}$ of nucleotides (Table 2). The length of these assembled unigenes ranged from 200 to $2,000 \mathrm{bp}$. The overall length distribution and GC content of the spliced unigenes are presented in Additional file 1.
Table 2 Length distribution of the transcripts and unigenes clustered from the de novo assembly

\begin{tabular}{lll}
\hline Category & Transcript & Unigene \\
\hline All (>=200 bp) & 415,429 & 143,080 \\
$>=500 \mathrm{bp}$ & 273,632 & 56,929 \\
$>=1000 \mathrm{bp}$ & 181,131 & 30,038 \\
N50 & 1,747 & 1,239 \\
N90 & 497 & 277 \\
Total length & $236,414,577$ & $53,791,217$ \\
Max length & 14,995 & 14,995 \\
Min length & 201 & 201 \\
Average length & 1111.61 & 707.9 \\
\hline
\end{tabular}

Note: The N50 size is computed by sorting all transcripts from largest to smallest and by determining the minimum set of transcripts whose sizes total $50 \%$ of the entire transcript and unigene was the same; N90 was counted in the similar way.

Functional annotation of transcripts was mainly based on BLAST (Blastx tools) homology searches against various public protein databases (Table 3). Of the 143,080 non-redundant unigenes, 60,714 (42.43\%) showed a significant similarity to known proteins in the NR database and 33,992 (23.76\%) had significant hits in the SWISSPROT database. These results suggested an abundance of newly discovered unigenes. Gene Ontology (GO) analysis classified most of the 143,080 annotated unigenes into GO functional categories of biological process, cellular component, and molecular function (see Additional file 1).

\section{Identification of salt-responsive, differentially expressed unigenes}

To identify genes displaying significant expression changes during $\mathrm{NaCl}$ treatment, differentially expressed unigenes (DEUs) were analyzed by comparing 4- and 24-h libraries with the control library for both Nan Dan Ba Di Da Hua and Earlistaple 7 (Table 4). Salt-sensitive and salt-tolerant

Table 1 The data quality of mRNA-seq and the transcripts in two genotypes

\begin{tabular}{|c|c|c|c|c|c|c|c|}
\hline \multirow[t]{2}{*}{ Summary } & & \multicolumn{3}{|c|}{ Nan Dan Ba Di Da Hua } & \multicolumn{3}{|c|}{ Earlistaple 7} \\
\hline & & CK & $4 \mathrm{~h}$ & $24 \mathrm{~h}$ & $\overline{C K}$ & $4 \mathrm{~h}$ & $24 \mathrm{~h}$ \\
\hline Raw data & Read & $19,277,194$ & $12,479,678$ & $12,479,678$ & $17,969,500$ & $17,969,500$ & $21,602,254$ \\
\hline Raw data & Base & $1,927,719,400$ & $1,247,967,800$ & $1,247,967,800$ & $1,796,950,000$ & $1,796,950,000$ & $2,160,225,400$ \\
\hline Valid data & Read & 16,097034 & $10,492,532$ & $10,492,532$ & $15,026,640$ & $15,026,640$ & $18,100,020$ \\
\hline Valid data & Base & $1,497,064,827$ & $977,315,799$ & $977,315,799$ & $1,396,615,990$ & $1,396,615,990$ & $1,685,347,764$ \\
\hline Valid data & Average length & 93 & 93.14 & 93.14 & 92.94 & 92.94 & 93.11 \\
\hline Valid data & Valid ratio & $83.50 \%$ & $84.08 \%$ & $84.08 \%$ & $83.62 \%$ & $83.62 \%$ & $83.79 \%$ \\
\hline MapData & Number & $13,688,457$ & $12,721,295$ & $8,976,783$ & $10,560,871$ & $12,677,667$ & $15,465,674$ \\
\hline MapData & Data\% & $85.04 \%$ & $84.29 \%$ & $85.55 \%$ & $84.58 \%$ & $84.37 \%$ & $85.45 \%$ \\
\hline ExpTranscript & Number & 374,204 & 372,440 & 358,476 & 367,228 & 374,490 & 381,094 \\
\hline ExpTranscript & Sum. & 809418.58 & 803094.61 & 785100.61 & 819293.67 & 805543.43 & 792193.63 \\
\hline ExpTranscript & Transcript\% & $87.97 \%$ & $87.56 \%$ & $84.28 \%$ & $86.33 \%$ & $88.04 \%$ & $89.59 \%$ \\
\hline
\end{tabular}


Table 3 The numbers and distribution rate of unigenes in the databases of NR, SWISS-PROT, TrEMBL, CDD, PRAM, KOG and KEEG

\begin{tabular}{llllllll}
\hline Unigene no. & NR & SWISS-PROT & TrEMBL & CDD & PFAM & KOG & KEGG \\
\hline 143080 & 60,714 & 33,992 & 62,473 & 29,641 & 54,865 & 17,958 & 20,241 \\
& $42.43 \%$ & $23.76 \%$ & $43.660 \%$ & $20.72 \%$ & $38.35 \%$ & $12.55 \%$ & $14.15 \%$ \\
\hline
\end{tabular}

Note: Comparison of the all unigenes with public databases like Non-redundant nucleotide database (NR), SWISS-PROT ((UniProt), TrEMBL and PFAM, KOG and KEGG, respectively, functional annotation was done through gene similarity $>30 \%$ and e-value $<1 \mathrm{e}^{-5}$.

genotypes showed very similar expression patterns. As the salt treatment duration lengthened, an increasing number of gene expression changes were observed in both genotypes. In addition, the number of up-regulated genes in the salt-tolerant genotype (Earlistaple 7) was more than in salt-sensitive genotype.

Transcription factors (TFs) play key roles in modulating the acclimation response of plants to severe environments. In our study, $2.2 \%(3,172)$ of the 143,080 unigenes encoding TF family members in Earlistaple 7 and Nan Dan Ba Di Da Hua were responsive to 4- and 24-h treatments with $200 \mathrm{mM} \mathrm{NaCl}$. These unigenes were classified into 52 TF families and 1 group of putative TFs (see Additional file 2), including several key regulatory gene families involved in response to abiotic and biotic stress, such as AP2-EREBP (311), ARF (106), bHLH (254), bZIP (104), C2C2-Dof (90), C2H2 (94), DBP (163), GRAS (130), HB (136), MYB (225), NAC (170), and WRKY (222). Among these TFs, AP2-EREBP, MYB, NAC, and WRKY members unigenes were mostly up-regulated under salt stress, while bHLH and $\mathrm{C} 2 \mathrm{C} 2$ families were represented by a balanced number of up- and down-regulated members (see Additional file 2). Many identified TFs belonged to AP2/EREBP, and most were involved in stress response. These results imply that AP2/EREBP family members play an important role in regulation of salt stress tolerance in G. hirsutum. MYB, NAC, and WRKY were also highly enriched during 4- and 24-h salt stress (Figure 2).

\section{Dynamic change analysis of co-expressed unigenes}

To understand dynamic expression patterns of DEUs under salt stress at different time points, the various gene expression modes in Earlistaple 7 were identified. The most representative significantly DEU expression mode was then selected for further analysis. To uncover the mechanisms underlying salt sensitivity in Nan Dan Ba Di
Da Hua, we used Short Time-series Expression Miner (STEM) software to analyze abundance changes of differentially expressed TF unigenes and other significant functional unigenes filtered by different genotypes at different time points. The analytical procedure used is outlined in Additional file 3 .

The functional enrichment of genes with distinct patterns (among co-expressed gene clusters) in Earlistaple 7 was also analyzed using STEM software. Seven significant expression profiles $(P<0.001)$ were identified. As shown in Figure 3A, significantly different profiles were represented by different background colors (red or green). According to the background color, the seven most representative expression patterns were divided into two clusters, one containing four and one containing three profiles. Unigenes within the same cluster were considered to be co-expressed genes following the same expression pattern. Differentially expressed unigenes of Earlistaple 7 at two time points ( 4 and $24 \mathrm{~h}$ ) were selected from the two clusters and subjected to GO category gene enrichment analysis. The two unigene set comparisons (Earlistaple 7 at 4 vs. $0 \mathrm{~h}$ [E4/E0] and Earlistaple 7 at 24 vs. $0 \mathrm{~h}[\mathrm{E} 24 / \mathrm{E} 0])$ that were enriched $(P<0.01)$ for certain GO categories (at a level of 3 or below in the GO hierarchy) are shown in Figure 3B. The 18 most abundant GO terms into which E4/E0 and E24/E0 unigene sets were distributed included many abiotic stress-related categories, such as "transferase activity", "nucleic acid binding", "nucleotide binding", and "ion binding". In addition, several GO terms showed significant differences in enrichment between 4- and 24-h salt treatments, with "Response to stress", "regulation of biological process", "transcription factor activity", and "structural constituent of ribosome" most prominent. It is increasingly clear that stress and metabolic signaling networks interact, and that this interaction is important in plant response to abiotic stresses

Table 4 The numbers of all differentially expressed unigenes (DEUs) at different salt-stressed time points of two genotypes

\begin{tabular}{|c|c|c|c|c|}
\hline \multirow[t]{2}{*}{ Items } & \multicolumn{2}{|c|}{ Nan Dan Ba Di Da Hua } & \multicolumn{2}{|l|}{ Earlistaple 7} \\
\hline & 0 versus $4 \mathrm{~h}$ & 0 versus $24 h$ & 0 versus $4 \mathrm{~h}$ & 0 versus $24 \mathrm{~h}$ \\
\hline Total & 66,803 & 71,267 & 62,928 & 71,311 \\
\hline Up-regulated & $32,789(49 \%)$ & $31,423(44 \%)$ & $33,790(54 \%)$ & $40,131(56 \%)$ \\
\hline Down-regulated & $34,014(51 \%)$ & $39,844(56 \%)$ & $29,138(46 \%)$ & $31,180(44 \%)$ \\
\hline
\end{tabular}

Note: Number of DEUs $\left(P<0.05\right.$ and $\mid \log _{2}$ Ratio| $\left.\geq 1\right)$ under $\mathrm{NaCl}$ stress for 4-h and 24-h as compared to their respective control samples. 


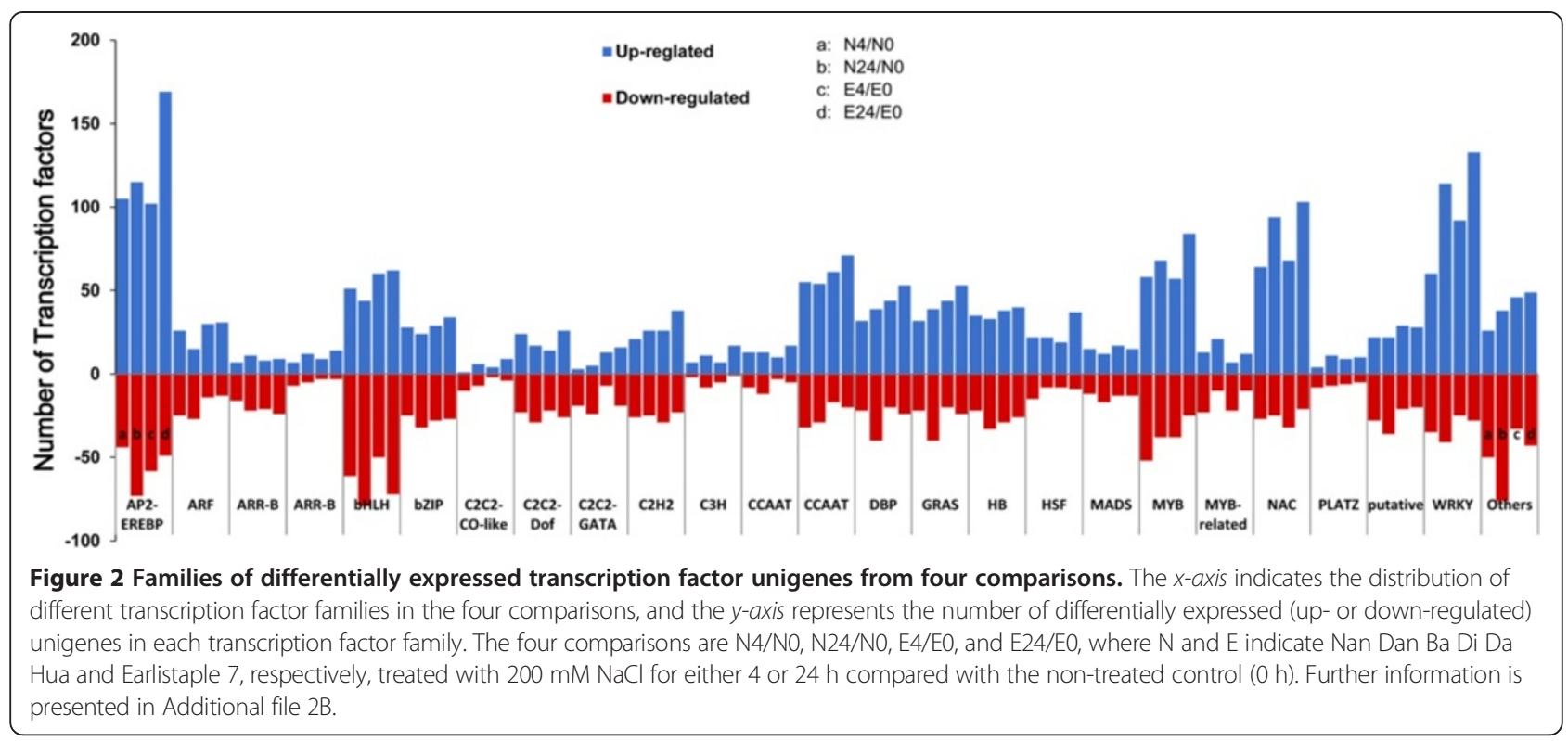

[51]. The remaining clusters were not enriched for GO categories because of the many unannotated genes in the transcriptome, suggesting that numerous pathways involved in stress tolerance are yet to be revealed.

All differentially co-expressed unigenes were classified into three categories according to the significantly different expression patterns identified (Table 5). We also performed a STEM cluster analysis of all DEUs from the Earlistaple 7 genotype (Table 6), which revealed six distinct groups: early-stage up-regulated genes (Group I), late-stage up-regulated genes (Group II), continuously upregulated genes (Group III), early-stage down-regulated genes (Group IV), late-stage down-regulated genes (Group $\mathrm{V}$ ), and continuously down-regulated genes (Group VI). GO categories were assigned to DEUs in the six data sets (i.e., unigenes expressed in both genotypes at 4, 24, and both 4 and $24 \mathrm{~h}$, and unigenes specifically expressed in Earlistaple 7 at 4,24 , and both 4 and 24 h) (Figure 4). In the biological process category, $\mathrm{GO}$ classification of DEUs in the three data sets of expressed unigenes that were common to both genotypes at 4, 24, and both 4 and $24 \mathrm{~h}$ was similar, but the number of commonly expressed unigenes between the two genotypes at $4 \mathrm{~h}$ was less than the number at 24 and both 4 and $24 \mathrm{~h}$ (Figure $4 \mathrm{~A}$ ). In the molecular function category, the four most abundant subcategories in the three data sets of Earlistaple-7-specific unigenes were nucleic acid binding, nucleotide binding, transferase activity, and ion binding (Figure 4B).

\section{Analysis of salt stress-responsive TFs and other salt tolerance-related functional genes}

Although expression patterns of many unigenes were similar between Earlistaple 7 and Nan Dan Ba Di Da Hua, $78.2 \%$ of salt-responsive unigenes identified in Earlistaple
7 were more or less differentially expressed (Nominal false discovery rate $($ FDR $)<0.001)$ in Nan Dan Ba Di Da Hua at one or more time points (Table 5). TFs are important regulators of gene expression. The related the expression abundance and annotation information of TF unigenes in the six data sets by STEM analysis were obtained (see Additional file 2C-H).

An overlap was only observed in TF unigenes responsive to $\mathrm{NaCl}$ stress between Earlistaple 7 and Nan Dan Ba Di $\mathrm{Da}$ Hua at the 4-h time point, with 55 TFs transcriptionally up-regulated and 60 down-regulated (Table 6). These differentially expressed TFs belonging to AP2-EREBP family were followed in abundance by WRKY, NAC, MYB, and $\mathrm{C} 2 \mathrm{H} 2$ under 4- and 24-h $\mathrm{NaCl}$ treatment (see Additional file $2 \mathrm{C}-\mathrm{E}$ ). It should be noted that all of these DEUs were expressed in the salt-tolerant genotype Earlistaple 7, but were repressed, weakly induced, or not induced at all in salt-sensitive Nan Dan Ba Di Da Hua (see Additional file 2I and Figure 3C). Some of these weakly/non-induced genes, such as GhNAC4, GhNAC5, GhWRKY2, GhERF5, and $G h D R E B 1 L$, play very important roles in salt tolerance of cotton (Table 7).

Under $\mathrm{NaCl}$ stress, most of the salt-responsive TF unigenes exhibited genotype specificity. We identified 129 TF unigenes whose expressions were specific to Earlistaple 7 , of which 85 were up-regulated and 44 were suppressed under salt stress (see Additional file $2 \mathrm{~F}-\mathrm{H}$ ). These 129 TF unigenes, which corresponded to $1.6 \%$ of the 8,127 salt-responsive genes of Earlistaple 7, were only expressed during either early or late stages of salt stress. We note that some TF unigene families expressed under both 4- and 24-h salt stress were identical to the above-mentioned TF families commonly expressed in both genotypes, but the expressed TF was different. The 


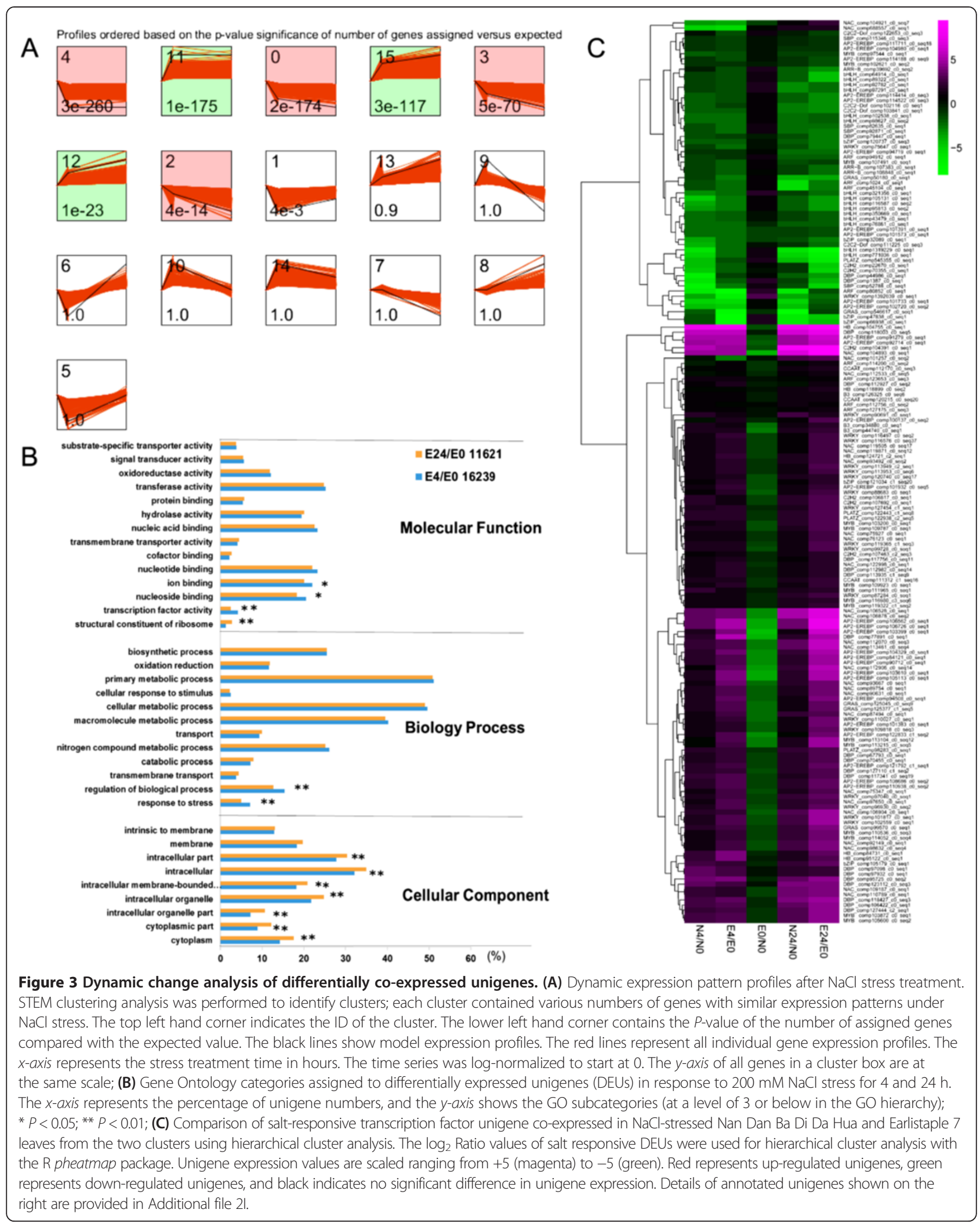


Table 5 Summary of the numbers of differentially co-expressed unigenes from the cluster 1 and cluster 2 by STEM analysis

\begin{tabular}{|c|c|c|c|c|c|}
\hline & Categories & $\mathrm{ck}, 4 \mathrm{~h}$ & ck, $24 \mathrm{~h}$ & $c k, 4 h, 24 \mathrm{~h}$ & Total \\
\hline$\overline{N-E ~ c o m m o n ~}$ & & 16,180 & 24,137 & 32,602 & $72,919(78.2 \%)^{d}$ \\
\hline \multirow[t]{2}{*}{ unigenes number ${ }^{a}$} & Cluster 1 & $3,459(55)^{c}$ & $5,311(121)$ & $5,051(315)$ & $13,721(491)$ \\
\hline & Cluster 2 & $4,102(60)$ & $7,069(105)$ & $7,922(163)$ & $19,193(328)$ \\
\hline E-specific & & 5,619 & 6,754 & 7,918 & $20,291(21.8 \%)$ \\
\hline \multirow[t]{2}{*}{ Unigenes number ${ }^{\mathrm{b}}$} & Cluster 1 & $997(15)$ & $1,365(40)$ & $1,095(30)$ & $3,457(85)$ \\
\hline & Cluster 2 & $1,331(27)$ & $1,394(8)$ & $1,541(9)$ & $4,266(44)$ \\
\hline All & & 21,799 & 30,891 & 40,420 & 93,210 \\
\hline \multirow[t]{2}{*}{ Unigene numbers } & Cluster 1 & $4,456(70)$ & $6,6767(161)$ & $6,146(345)$ & $17,178(576)$ \\
\hline & Cluster 2 & $5,433(87)$ & $8,463(113)$ & $9,463(172)$ & $23,339(372)$ \\
\hline
\end{tabular}

Note: ${ }^{\mathrm{a}} \mathrm{N}-\mathrm{E}$ common unigenes means the common differentially expressed unigenes between Nan Dan Ba Di Da Hua and Earlistaple 7.

${ }^{b}$ E specific unigenes means the specific DEUs expressed only in the Earlistaple 7.

cNumbers in parentheses indicate the number of TF unigenes.

${ }^{\mathrm{d}}$ The percentage of differentially expressed unigenes in both genotypes before STEM analysis.

expression trend of the WRKY TF unigene (homologous to PtWRKY25) was different at the two time points-decreasing at the beginning and then increasing, while that of others, such as AP2-EREBP, MYB, HB, NAC, WRKY, and bHLH family members, experienced a continual rise.

Many other salt tolerance-related unigenes encoding transporters, $\mathrm{Ca}^{2+}$ binding protein, heat-shock proteins (HSPs), detoxificants, and dehydration-response proteins were differentially expressed under 4- and 24-h salt stress (see Additional file 4). Several differentially expressed genes encoded transporters contributing to the reestablishment of ionic and osmotic homeostasis under salt stress, such as $\mathrm{ABC}$ transporters (i.e., $\mathrm{ABC}$ transporter $\mathrm{B}, \mathrm{C}$, and $\mathrm{G}$ family members), sodium/hydrogen exchanger (NHX1), ATPases $\left(\mathrm{Ca}^{2+}\right.$-ATPase, $\mathrm{H}^{+}$-ATPase, and $\mathrm{V}$-type $\mathrm{H}^{+}$-transporting ATPase), potassium transporter, and nitrate transporter. Genes encoding some $\mathrm{Ca}^{2+}$ sensors, namely CML and CPK, and one CBL-interacting protein kinase (CIPK) gene were identified in the different libraries. Two cotton CBL genes (GhCBL1 and GhCBL8) were highly expressed under 4- and/or 24-h salt stress in
Earlistaple 7. Unigenes encoding CDPK and CIPK members were up-regulated under 4- and/or 24-h salt stress in both genotypes. Genes from several HSP families (e.g., HSP20, HSP70, Hsp90, and DanJ) as well as genes related to reactive oxygen species (ROS) scavenging and detoxification (CAT, POD, GPX, ALDH, and $\mathrm{RBOH}$ ) and dehydration response (LEAs and RING/U-box domaincontaining protein/XERICO) were highly up-regulated at different salt-treatment time points either in Earlistaple 7 or Nan Dan Ba Di Da Hua (see Additional file 4). Finally, genes involved in salt-stress tolerance pathways, such as CDPK, starch and sucrose metabolism, carbohydrate metabolism, and MAPK signal pathways, were found to be induced at different time points. These identified unigenes are potentially salt-induced genes and may serve as a supplementary resource for salt-tolerance gene mining.

To further validate the results of mRNA sequencing, 26 transcripts were randomly selected along with their specific primers for quantitative real-time PCR (qRT-PCR) analysis (see Additional file 5A). Eleven of the 26 tested transcripts had expression patterns identical to those

Table 6 The classification and expression pattern of the identified differentially co-expressed unigenes

\begin{tabular}{|c|c|c|c|c|c|c|}
\hline Groups & I ck,4 h & II ck,24 h & III ck,4 h,24 h & IV ck,4 h & V ck,24 h & Vl ck,4 h,24 h \\
\hline Expression pattern & $\ldots$ & & & ........... & & \\
\hline Definition & $\begin{array}{l}\text { Early induced } \\
\text { up-regulated }\end{array}$ & $\begin{array}{l}\text { Late induced } \\
\text { up-regulated }\end{array}$ & $\begin{array}{l}\text { Continuous induced } \\
\text { up-regulated }\end{array}$ & $\begin{array}{l}\text { Early induced } \\
\text { down-regulated }\end{array}$ & $\begin{array}{l}\text { Late induced } \\
\text { down-regulated }\end{array}$ & $\begin{array}{l}\text { Continuous induceo } \\
\text { down-regulated }\end{array}$ \\
\hline $\mathrm{N}-\mathrm{E}$ common unigenes ${ }^{\mathrm{a}}$ no. & $3,459(55)^{c}$ & $5,311(121)$ & $5,051(315)$ & $4,102(60)$ & $7,069(105)$ & $7,922(163)$ \\
\hline E specific unigenes ${ }^{\mathrm{b}}$ no. & $997(15)$ & $1,365(40)$ & $1,095(30)$ & $1,321(27)$ & $1,394(8)$ & $1,541(9)$ \\
\hline
\end{tabular}

${ }_{\mathrm{a}}^{\mathrm{N}}$-E common unigenes means the Nan Dan Ba Di Da Hua and Earlistaple 7 are common to differentially co-expressed unigenes.

${ }^{b} \mathrm{E}$ special unigenes means the differentially co-expressed unigenes expressed only in the Earlistaple 7 genotype.

${ }^{\mathrm{c}}$ Numbers in parentheses indicate the number of TF unigenes. 


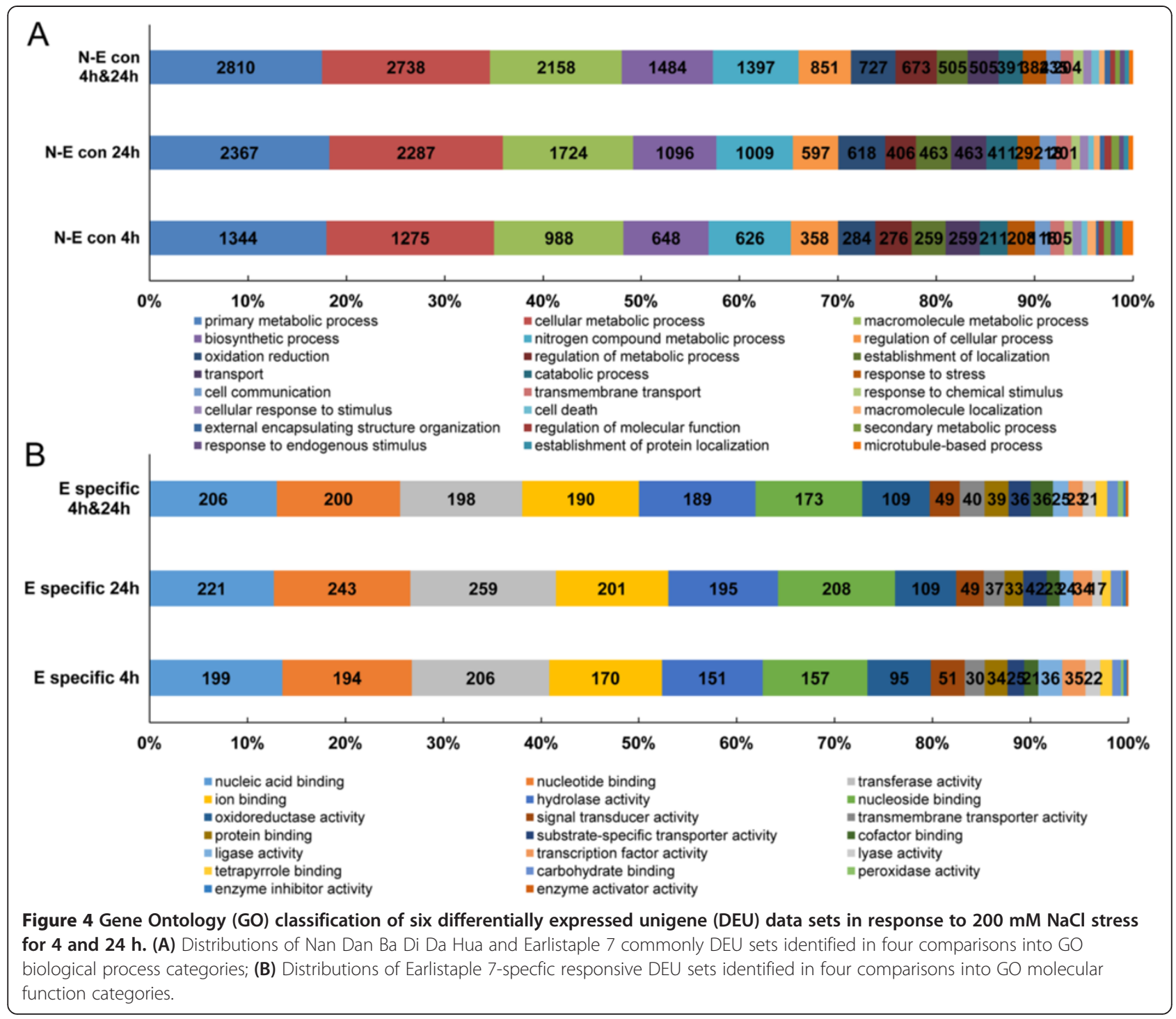

observed in the mRNA-seq experiments (Figure 5A). Expression levels measured by qRT-PCR were strongly correlated with those of DEUs identified by mRNA-seq $(r=0.793$ and 0.877 under 4 - and $24-\mathrm{h}$ salt stress, respectively), demonstrating the reliability of the mRNA-seq results (Figure 5B and Additional file 5D).

\section{Deep sequencing of small RNAs and identification of conserved miRNAs under salt stress}

To characterize differences between Nan Dan Ba Di Da Hua and Earlistaple 7 at the miRNA regulatory level and to identify conserved miRNAs in cotton under salt stress, we constructed and analyzed six small RNA libraries of the two genotypes. A total of 48,585,151 sequence reads ranging in length from 17 to $35 \mathrm{nt}$ were generated. After removing adapter sequences, low-quality tags, and reads shorter than $18 \mathrm{nt}$, we obtained 22,681,645 clean reads, which corresponded to more than 2 million unique sequences for each sample (see Additional file 6A). As shown in Additional file $6 \mathrm{~B}$, most small RNAs were 20-24 nt in size; the 24-nt class was the most heavily represented (50.56\%) in the Nan Dan Ba Di Da Hua small RNA control library, followed by 23-nt (7.37\%), 21-nt (10.27\%), and 22-nt classes (7.14\%).

The six libraries of clean reads were compared against tRNAdb, SILVA rRNA, and NONCODE v3.0 databases, allowing for mismatches. Detailed results of this comparison are shown in Table 8. After annotation and removal of non-coding small RNAs, 320 conserved miRNAs were identified and grouped into 37 miRNA families, with those belonging to unknown families designated by "NA" Comparison of the six libraries against the miRbase19.0 database, which contains 39 known ghr (G. hirsutum) miRNAs, identified 21 conserved miRNAs belonging to 17 families, including three families that are cotton-specific: miR2948 (ghr-miR2948-5p), miR2949 (ghr-miR2949a-5p, 
Table 7 The specific transcription factor unigenes response to salt stress in Earlistaple 7

\begin{tabular}{|c|c|c|c|c|c|}
\hline \multirow[t]{2}{*}{ Unigene ID } & \multirow[t]{2}{*}{ Accession } & \multirow[t]{2}{*}{ Annotation } & \multirow[t]{2}{*}{ E-value } & \multicolumn{2}{|c|}{ Earlistaple 7 Log2 ratio } \\
\hline & & & & $4 \mathrm{~h}$ & $24 \mathrm{~h}$ \\
\hline comp1995_c0_seq1 & Q9FWX2 & NAC domain-containing protein 7 & $7 \mathrm{E}-51$ & -2.00 & -1.52 \\
\hline comp105823_c0_seq1 & Q39261 & Zinc finger protein 2 & $9 \mathrm{E}-22$ & -1.63 & -1.39 \\
\hline comp100893_c1_seq1 & Q97WM9 & AP2/ERF and B3 domain transcription factor RAV & $8 \mathrm{E}-78$ & -1.01 & -1.65 \\
\hline comp109426_c0_seq4 & Q9FLX8 & Probable WRKY transcription factor 27 & $9 \mathrm{E}-58$ & -0.56 & -2.14 \\
\hline comp1104709_c0_seq1 & Q39265 & Zinc finger protein 6 & $2 \mathrm{E}-25$ & 0.00 & -1.53 \\
\hline comp534425_c0_seq1 & B9SUH9 & CCAAT-binding transcription factor. Putative & $2 \mathrm{E}-11$ & 0.00 & -1.58 \\
\hline comp94181_c0_seq1 & Q688R3 & Zinc finger $\mathrm{CCCH}$ domain-containing protein 33 & $1 \mathrm{E}-16$ & 1.05 & 2.14 \\
\hline comp105064_c1_seq2 & Q9XEE6 & Zinc finger $\mathrm{CCCH}$ domain-containing protein 29 & $6 \mathrm{E}-79$ & 1.07 & 2.10 \\
\hline comp77518_c0_seq1 & E2FGB5 & Homeodomain-leucine zipper protein HD2 GhHB2 & $1 \mathrm{E}-53$ & 1.92 & 3.29 \\
\hline comp103962_c0_seq1 & Q6VY01 & Putative dehydration responsive GhDREB1A & $3 \mathrm{E}-53$ & 2.26 & 3.62 \\
\hline comp87977_c0_seq1 & Q9LR65 & Probable protein phosphatase $2 \mathrm{C} 1$ & $1 \mathrm{E}-69$ & 2.47 & 2.35 \\
\hline comp64928_c0_seq1 & B9GYM6 & Ethylene-responsive transcription factor ERF105 & $6 \mathrm{E}-07$ & & 2.18 \\
\hline comp97922_c0_seq1 & Q9XEE6 & Zinc finger CCCH domain-containing protein 29 & $4 \mathrm{E}-91$ & & 2.08 \\
\hline comp96688_c0_seq1 & Q8LCG7 & Nuclear transcription factor Y subunit C-2 NFYC2 & $3 E-26$ & & 1.98 \\
\hline comp72703_c0_seq1 & Q9SV15 & Probable WRKY transcription factor 11 & $7 \mathrm{E}-27$ & & 1.98 \\
\hline comp108958_c0_seq1 & B9SRT4 & WRKY transcription factor. Putative & $1 \mathrm{E}-65$ & & 1.89 \\
\hline comp74963_c0_seq2 & Q9XJ60 & MADS-box transcription factor 50 & $1 \mathrm{E}-30$ & & 1.85 \\
\hline comp106969_c0_seq1 & Q6X7J9 & WUSCHEL-related homeobox 4 & $5 E-60$ & & -1.2 \\
\hline comp67684_c0_seq1 & Q9FJV5 & Probable transcription factor KAN4 & 3E-09 & & -1.48 \\
\hline comp81996_c0_seq1 & A4L9W4 & Auxin response factor 3 GhARF3 & $9 \mathrm{E}-16$ & & -2.14 \\
\hline comp114981_c0_seq2 & Q9FHH8 & Zinc finger protein CONSTANS-LIKE 5 & $3 \mathrm{E}-23$ & & -2.15 \\
\hline comp426377_c0_seq1 & A9PL22 & Homeobox protein GhHB1 & $1 \mathrm{E}-58$ & & -2.88 \\
\hline comp79059_c0_seq1 & Q9SK55 & NAC domain-containing protein 42 & $3 \mathrm{E}-77$ & -4.11 & \\
\hline comp60998_c0_seq1 & Q681X4 & Zinc finger protein ZAT5 & $6 \mathrm{E}-16$ & -2.55 & \\
\hline comp71059_c0_seq1 & O80933 & Scarecrow-like protein 9 & $5 E-41$ & -2.45 & \\
\hline comp107981_c0_seq1 & B9SMN9 & Transcription factor. Putative & $7 \mathrm{E}-33$ & -2.41 & \\
\hline comp98436_c0_seq1 & D3XFF8 & TT2 like MYB transcription factor & $2 \mathrm{E}-77$ & -2.26 & \\
\hline comp398_c0_seq1 & 004291 & Homeobox-leucine zipper protein ATHB-14 & $3 \mathrm{E}-25$ & -2.15 & \\
\hline comp108793_c0_seq1 & Q8LAP8 & Dof zinc finger protein DOF4.6 & $2 \mathrm{E}-38$ & -2.1 & \\
\hline comp2403_c0_seq1 & Q9SB61 & ZF-HD homeobox protein At4g24660 & $2 \mathrm{E}-41$ & -1.84 & \\
\hline comp55428_c0_seq1 & G7JVA8 & Zinc finger protein-like Ser/Thr protein kinase-like protein & $5 \mathrm{E}-75$ & -1.44 & \\
\hline comp69223_c0_seq1 & Q9LHJ9 & Probable protein phosphatase $2 C 38$ & $2 \mathrm{E}-142$ & 1.63 & \\
\hline comp67435_c0_seq1 & B9RA11 & Transcription factor. Putative & $2 \mathrm{E}-26$ & 1.71 & \\
\hline comp60938_c0_seq1 & E6Y3E2 & WRKY transcription factor PmWRKY110 (Fragment) & $1 \mathrm{E}-11$ & 1.96 & \\
\hline comp798487_c0_seq1 & Q4ZJA9 & BZIP-like protein Gossypium hirsutum & $2 \mathrm{E}-19$ & 2.5 & \\
\hline
\end{tabular}

ghr-miR2949a-3p, and ghr-miR2949b), and miR3476 (ghr-miR3476-5p) (see Additional file 7).

The number of identified miRNAs and the abundance of miRNA families are listed in Additional file 6C-D and Additional file 7 . The reads for each miRNA family were normalized as "reads per million mapped reads" (RPM). Among miRNA families, miR166 exhibited the highest abundance $(3,909,410$ RPM). Other highly expressed families, including miR396, miR482, miR159, miR1697, miR394, and miR156, were represented by approximately 100,000 RPM, whereas miR319, miR2118, miR858, miR828, miR829 and miR1310 families had less than 100 RPM. Differences among miRNA families were also revealed by the number of members they contained (see Additional file 6D and Additional file 7). miR166 was the largest family, with 29 members, while miR159, miR396, miR171, and miR156 


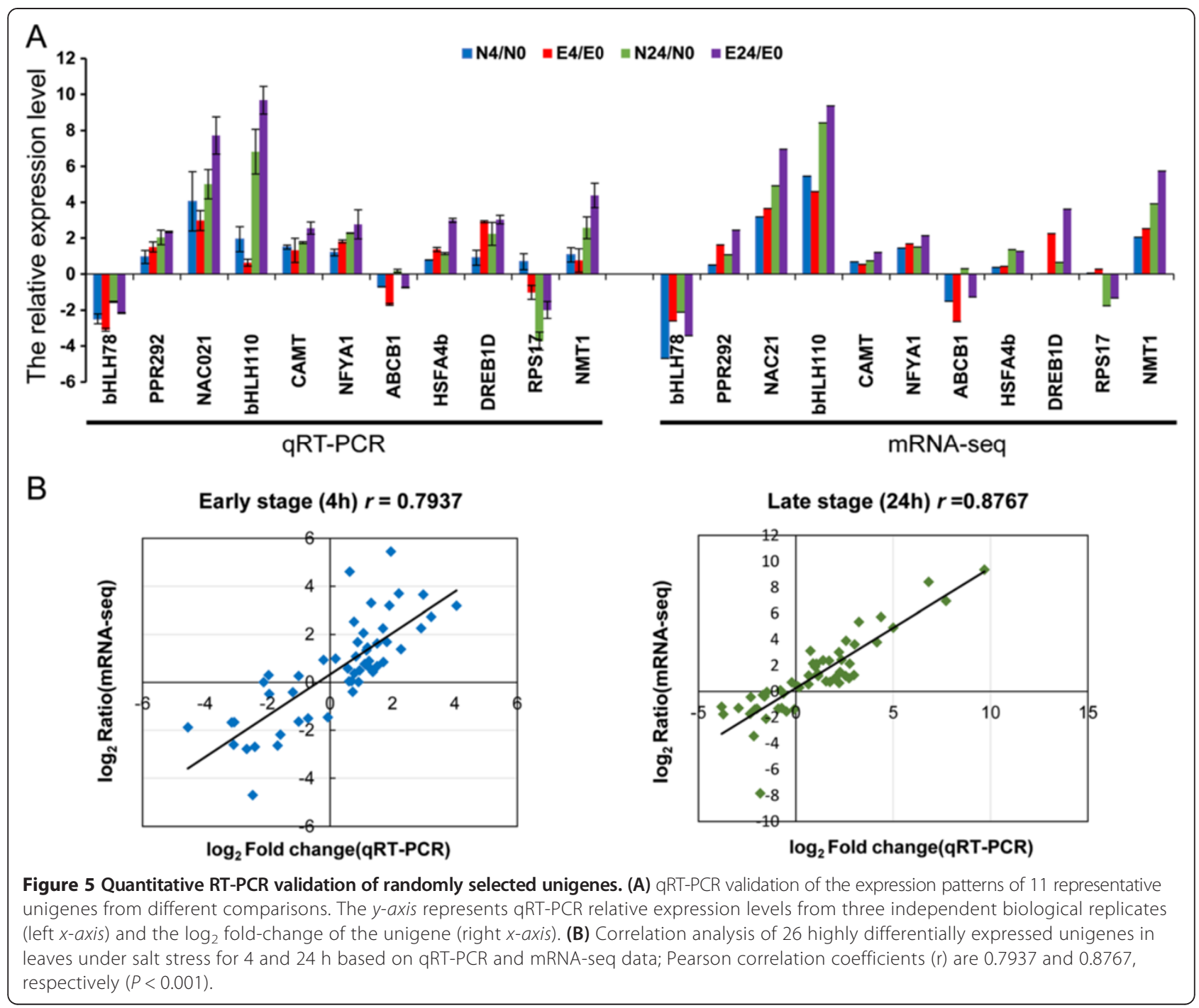

Table 8 Distribution of sequence reads among different RNA categories in six libraries of cotton

\begin{tabular}{lllllllll}
\hline & Sample & All & rRNA & snoRNA & tRNA & snRNA & miRNA & Unanotation \\
\hline Unique sRNA number & N0 & 440,466 & 32574.5 & 153.5 & 283 & 235 & $626(0.14 \%)$ & 406,594 \\
& N4 & 431,000 & 36,940 & 166 & 297 & 249 & $650(0.15 \%)$ & 392,698 \\
& N24 & 339,460 & 36,430 & 101 & 272 & 257 & $508(0.15 \%)$ & 301,892 \\
& E0 & 237,949 & 25,002 & 94 & 176 & 107 & $479(0.20 \%)$ & 212,091 \\
& E4 & 365,569 & 34,035 & 133 & 198 & 253 & $449(0.12 \%)$ & 330,501 \\
Total sRNA number & E24 & 807,866 & 33,293 & 64 & 134 & 90 & $376(0.05 \%)$ & 773,909 \\
& N0 & $4,226,267$ & $1,073,632$ & 1705 & 3596 & 1165 & $320,413(7.58 \%)$ & $2,825,756$ \\
& N4 & $4,208,092$ & $1,207,710$ & 1812 & 3603 & 1065 & $335,427(7.97 \%)$ & $2,658,475$ \\
& N24 & $4,821,011$ & $1,395,936$ & 1782 & 3923 & 1656 & $379,404(7.87 \%)$ & $3,038,310$ \\
& E0 & $2,378,551$ & 626,216 & 813.5 & 2138 & 368 & $327,868(13.78 \%)$ & $1,421,148$ \\
& E4 & $2,999,177$ & 814,357 & 1250 & 2197 & 918 & $335,427(11.18 \%)$ & $1,845,028$ \\
& E24 & $4,048,547$ & 461,580 & 236 & 1702 & 234 & $73,340(1.81 \%)$ & $3,511,455$ \\
\hline
\end{tabular}


possessed 28, 26, 23, and 20 members, respectively. Different family members also displayed drastically different expression levels (see Additional file 7). In the miR156 family, for instance, RPM of ghr-miR156a was 308 in the control Nan Dan Ba Di Da Hua library, while the RPM of cca-miR156b was only 2.6.

We identified miRNAs that were differentially expressed $\left(P<0.05\right.$ and $\mid \log _{2}$ Ratio $\left.\mid \geq 1\right)$ between treatment and control (RPM of 4- and 24-h NaCl treatment compared with RPM of control) from 37 families (see Additional file 8). As shown in Figure 6A, the differentially expressed miRNAs were transcriptionally regulated at different time points in the two genotypes under salt stress. We detected both congruously and oppositely regulated miRNAs as well as those that were genotype-specific (Figure 6B and Additional file 8). Further analysis revealed that most differentially expressed miRNAs displayed different expression patterns between the two genotypes or the two time points (see Additional file 9A, B). These results demonstrate that miRNA expression changes in cotton under early-( $4 \mathrm{~h})$ and late-stage ( $24 \mathrm{~h})$ salt stress are similar, and that differential regulation of miRNAs may be responsible for the distinct salt sensitivities of the two cotton genotypes.

\section{Combined analysis of conserved miRNAs and their target genes under salt stress}

To discover salt tolerance-related miRNAs in cotton, we used the analytical approach shown in Additional file 9C. We uncovered 108 differentially expressed miRNAs and compared their expressions in the two genotypes (see Additional file 8E). At least one comparison (E4/E0 or E24/E0) showed significant changes at the $P<0.05$ level. By incorporating the transcriptome sequencing results into the comparisons E4/E0 and E24/E0 for all 108 differentially expressed miRNAs under salt stress, we detected 761 and 1,016 miRNA-target pairs, respectively. Generally, microRNAs negatively regulate the accumulation of their target mRNAs therefore when they are expressed in common tissue, their expression profiles should show negative correlation [34]. But [52] found an equal number of positively and negatively correlated miRNA/target pairs indicating that positive correlation is more frequent than previously thought [52]. In our research, we attempted to reveal the molecular regulation mechanism of salt tolerance in upland cotton, microRNA might played an important role during the response process. Therefore, the first step was to found out the correlationship between miRNA expression and target mRNA expression, we calculated Pearson correlation coefficients (PCCs) between miRNAs and their targets. The PCCs between all 761 and 1,016 miRNAs and their target pairs were -0.0362 and 0.0007 at $4-\mathrm{h}$ and 24-h in Earlistaple 7, respectively, indicating no correlations (Figure 7A, D). Then we found these miRNA were grouped into three categories: positively correlated, negatively correlated, and not correlated according to their target gene expression. It is obviously the no significant correlation between total miRNAs and their targets maybe result from the opposite effects of the positively and negatively correlation of the miRNA/target pairs. Therefore, we should separate the positively correlated and negatively correlated miRNA/gene pairs for further analysis based on the previous studies [52], The PCC and linear regression analysis of the positively and negatively correlated miRNA/ gene pairs were shown in Figure 7B, C, E, F. These results suggested that negative correlation is similar to the positive correlation and complex regulatory networks exist between miRNAs and their targets in cotton under salt stress. Finally, after excluding unannotated target unigenes from E4/E0 and E24/E0 comparisons, we respectively obtained 65 and 101 negative miRNA-target gene relationships to further analysis (see Additional files 9D and $10 \mathrm{~A}, \mathrm{C})$. When $\mathrm{GO}$ categories were assigned to these targets, six molecular function categories predominated; among these, "hydrolase activity", "nucleoside binding",
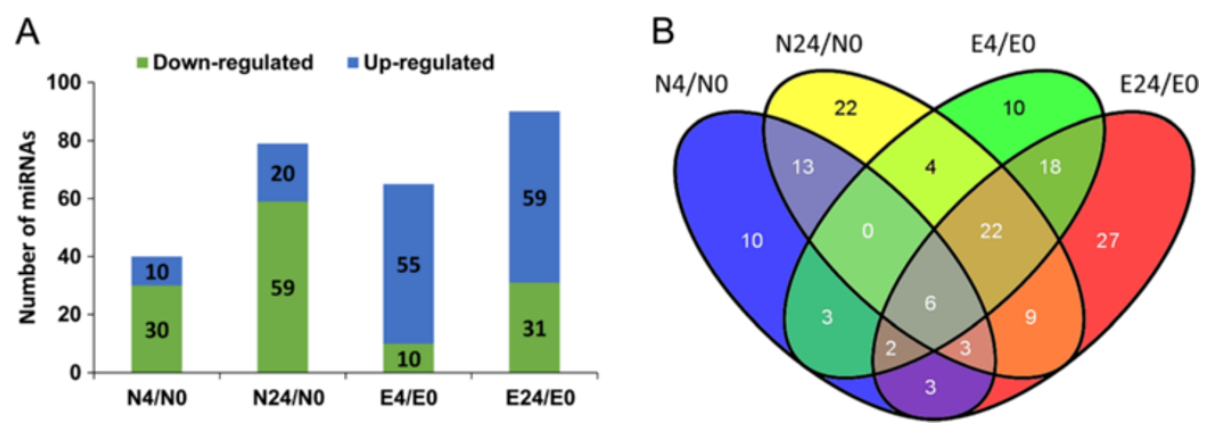

Figure 6 Abundances of differentially expressed conserved miRNAs identified in the two genotypes under 4- and 24-h salt stress. (A) Number of differentially expressed miRNAs $\left(P \leq 0.05\right.$ and $\mid \log _{2}$ Ratio| $\left.\geq 1\right)$ compared with their respective control samples; (B) Venn diagram illustrating the number of congruously and oppositely regulated miRNAs between Nan Dan Ba Di Da Hua and Earlistaple 7 in response to salt stress at various time points. 

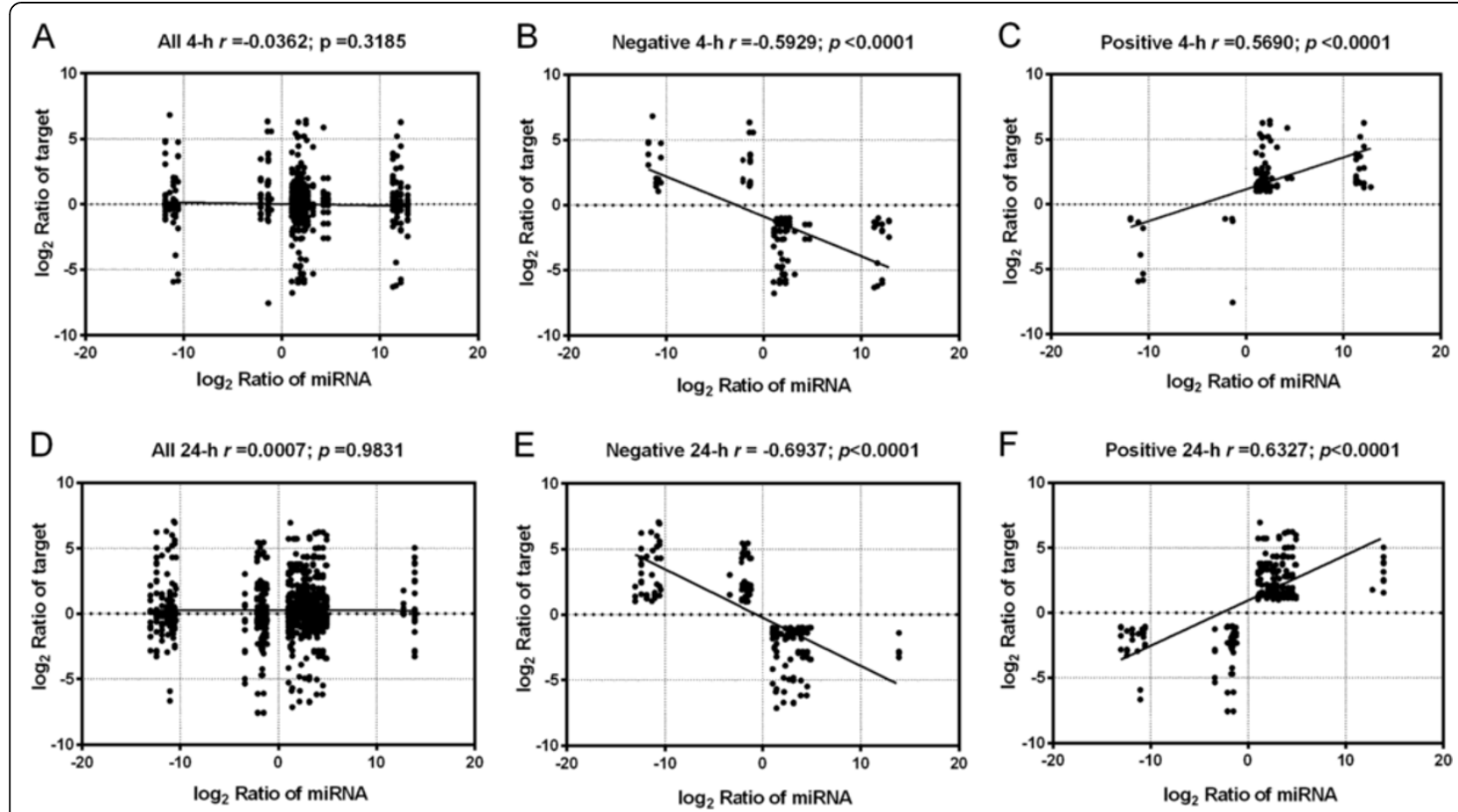

Figure $7 \mathrm{~A}$ combined view of correlated expression between a miRNA and its target in cotton under early- and late-stage salt stress. Pearson correlation coefficients (PCCS) between the expression of miRNAs and miRNA targets were calculated. Black circles represent miRNA and target pairs. PCCs are represented by the linear slope and are listed numerically, with $\mathbf{A}, \mathbf{B}$ and $\mathbf{C}$ indicating all, negative, and positive correlations for 4-h, respectively. The same means with $\mathbf{D}, \mathbf{E}$ and $\mathbf{F}$ for 24-h.

and "nucleotide binding" were the most highly represented. Fourteen biological processes were identified, the most frequent being "cellular metabolic process" and "primary metabolic process". With respect to cellular components, seven classes were uncovered, with "intracellular part" and "cell part" the most abundant (see Additional file 9E). These classifications suggest that miRNA targets associated with salt tolerance are primarily related to binding, catalytic activity, cellular metabolic, and other metabolic processes.

To elucidate the potential regulatory roles of miRNAs in cotton under salt stress, we analyzed differential expression profiles of 108 miRNAs in Earlistaple 7 and Nan Dan Ba Di Da Hua (see Additional file 11). According to the results of hierarchical cluster analysis, 57 of these representative conserved miRNAs exhibited significant differential expression during salt stress and were selected six clusters (a, b, c, d, e, f) were selected from a whole subbranch by hierarchical cluster analysis which indicated they presented a very similar expression pattern (Figure 8). To better understand the functions of the 57 representative conserved miRNAs and seven specifically expressed conserved miRNAs, we predicted their putative targets (see Additional file 11C,D,E).

We integrated information about the 64 conserved miRNAs into the E4/E0 and E24/E0 miRNA-target pair comparisons, which yielded 75 miRNA-target pairs for 40 conserved miRNAs from the six groups (Additional file 11C). The miRNA-target regulatory networks inferred at different time points $(4$ and $24 \mathrm{~h}$ ) are shown in Figure 9. Several conserved miRNAs (including miR156, 164, 166, $171,172,393,396$, and 397) were predicted to target potential TF genes such as those encoding squamosa promoter binding protein (SPL5), an NAC-domain transcription factor (NAC021), a bZIP protein $(A B I 5)$, an MYB transcription factor, Scarecrow-like protein (SCL6), AP2 domain-containing protein, Basic helix-loop-helix (bHLH78), an WRC domain (C3H), ethylene-responsive transcription factor (RAP2-7), and protein phosphatase $2 \mathrm{C}$ $(P 2 C 60)$. A total of 19 and 34 target genes related to 33 conserved miRNAs were induced in the salt-tolerant line Earlistaple 7 after 4 and $24 \mathrm{~h}$ of salt stress, respectively (Figure 9 and Additional file 11).

The expression patterns of nine conserved miRNAs were validated by stem-loop RT-PCR (Figure 10A and Additional file 5B). As expected, the stem-loop RT-PCR data showed a high degree of consistency with the expression profiles obtained by small RNA sequencing. These data also revealed that two miRNA targets were negatively regulated by their miRNAs. As shown in Figure 10B, at-miR393a expression increased between 4 and $24 \mathrm{~h}$ of salt treatment, whereas comp105131_c0_seq1 


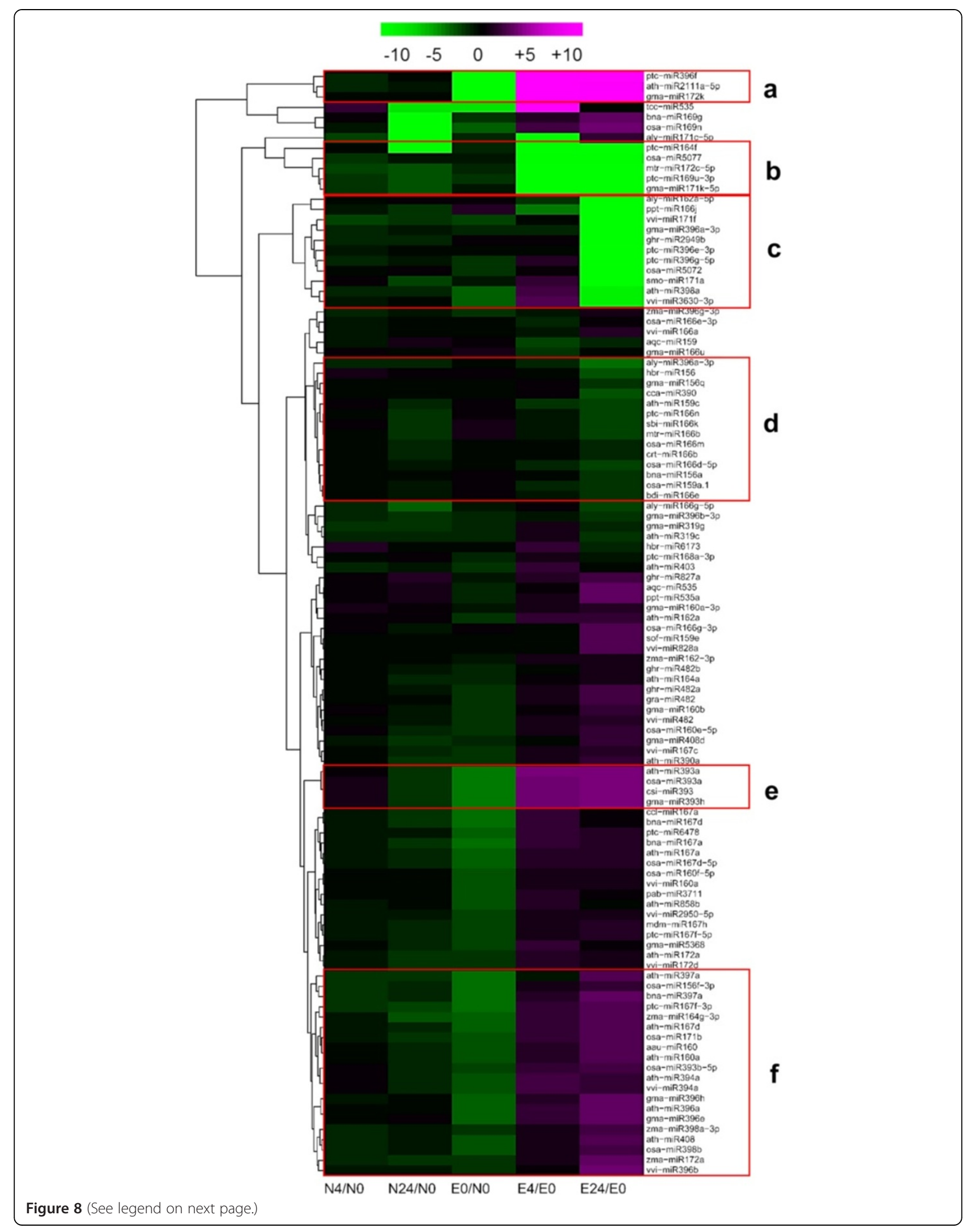


(See figure on previous page.)

Figure 8 Complete linkage hierarchical cluster analysis of 108 differentially expressed miRNAs in salt-tolerant Earlistaple 7. Log $_{2}$ Ratio was indicated on a color scale from magenta (high) to green (low). miRNA names are on the right side of the figure, and the five comparisons of both genotypes under 4- and 24-h of salt stress were at the bottom; The separated 57 representative conserved miRNAs were divided six clusters as following $\mathbf{a}, \mathbf{b}, \mathbf{c}, \mathbf{d}, \mathbf{e}, \mathbf{f}$ to further analysis. The miRNA name, log2 Ratio for each cluster are presented in Additional $11 \mathrm{~B}$.

(homologous to AtbHLH78) expression decreased, with its transcript levels negatively correlated with the accumulation of at-miR393a. Relative expression of ptc-miR164f was reduced to low levels at 4 and $24 \mathrm{~h}$ of salt treatment, while the expression level of its target gene (homologous to AtNACO21) remained unchanged, compared with the controls, during this period.

\section{Discussion}

Prerequisites for identification of candidate salt tolerance-related genes and miRNAs in cotton

Two main factors are responsible for salt stress-induced inhibition of plant growth: osmotic stress and ionic stress [50]. Different mechanisms control development at different time points in plants exposed to salinity. In this study, we monitored the physiological responses of two cotton genotypes with differing salt tolerances at two distinct phases. We found that these phases were characterized by increases or decreases of clusters of differentially expressed genes and miRNAs.

The positive relationship between water content and $\mathrm{Na}^{+}$concentration causes ions to accumulate in vacuoles, which lowers water potential, increases the driving force for water uptake, and increases turgor [53]. Maintenance of $\mathrm{K}^{+}$and $\mathrm{Na}^{+}$homeostasis is crucial under salt stress [54]. Our study results suggest that after being taken up by the roots, $\mathrm{Na}+$ is transported to shoots through the stems and the roots of salt-tolerant genotype Earlistaple 7

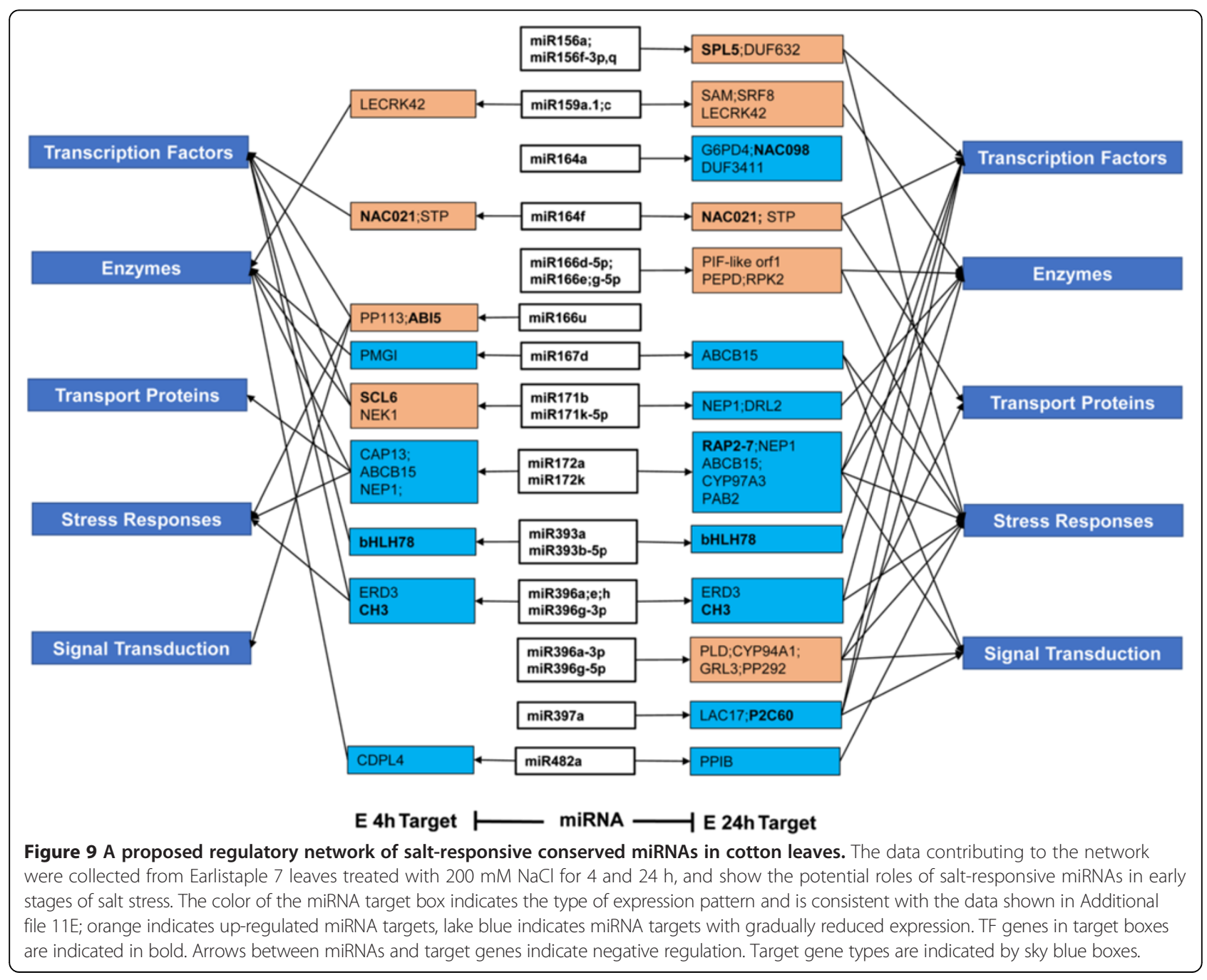




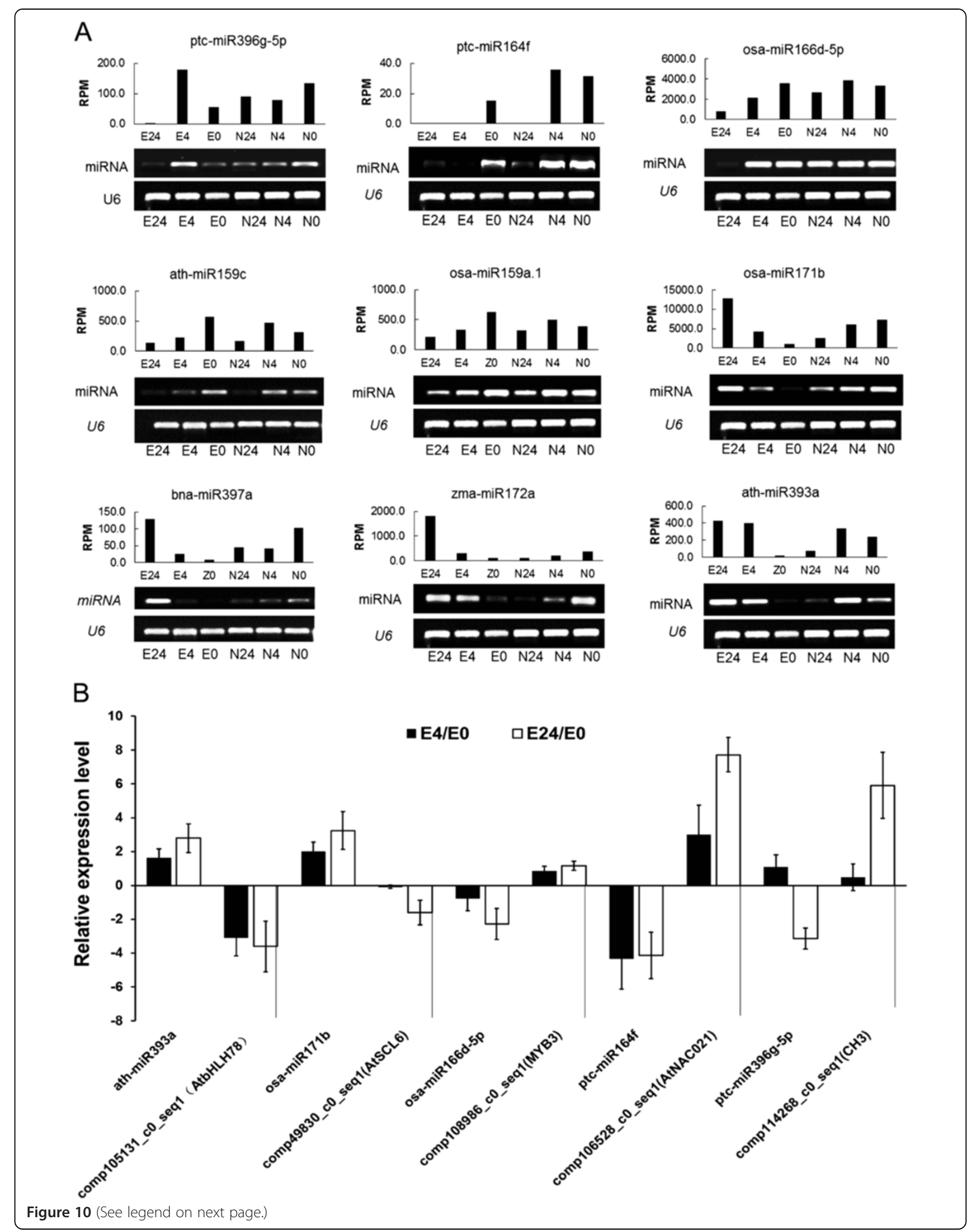


were able to retain more $\mathrm{Na}^{+}$than Nan Dan Ba Di Da Hua (Figure 1D, E). To avoid excessive ion accumulation in roots owing to continued $\mathrm{Na}^{+}$uptake, compensatory decreases in other cations occurred in the roots at $24 \mathrm{~h}$. Shoot leaves accumulated $\mathrm{Na}^{+}$in addition to the cations already present, with the tolerant genotype thereby experiencing the strongest ionic stress beginning at $24 \mathrm{~h}$ (Figure 1F).

By examining these dynamics, we gained insights into physiological responses of the $\mathrm{NaCl}$-acclimation process, which consisted of an initial dehydration phase in leaves $(0-4 \mathrm{~h})$ and subsequent $\mathrm{NaCl}$ accumulation (4-24 h) followed by restoration of osmotic homeostasis at a new ionic stress level $(24 \mathrm{~h})$ and either final adjustment to a steady ion balance or ion-induced damage (24-72 $\mathrm{h}$ and beyond). The timing of these adaptive responses differed between roots and leaves. In previous studies, either roots or leaves were used to identify differences between early and late transcriptional responses to salt treatment in cotton [31-34]. Our study of two genotypes with contrasting salt tolerances revealed that $\mathrm{Na}^{+}$accumulated mainly in shoots, and that significant physiological and morphological differences occurred at different time points under salt stress: $4 \mathrm{~h}$ (owing to dehydration stress) and $24 \mathrm{~h}$ (owing to ionic stress). These two important time points thus corresponded to the two typical phases of plant growth response to salt stress.

In some studies, genes differentially expressed in response to salt treatment have been identified using a single plant genotype [47-49]. Two or more genotypes with contrasting salt tolerance have only been used in a few reported investigations [46,55-57]. To identify genes responsible for salt tolerance, analysis of genotypes with similar genetic backgrounds and contrasting salt tolerances is preferable. Identification of common genes that are differentially expressed under stress conditions between tolerant and sensitive genotypes with different genetic backgrounds has been suggested as an alternative approach [58]. As pointed out in [58], it is impossible to isolate stress tolerance-related genes from stress-responsive ones unless transcriptional-level differences are compared between tolerant and sensitive genotypes under stress conditions [58]. Genes related to salt tolerance can thus be identified in genotypes with different genetic background and contrasting tolerances by comparative analysis of differentially expressed genes and miRNAs at different salt-treatment time points. We therefore used a pair of salt-tolerant and salt-sensitive genotypes in this study to identify differentially expressed genes and miRNAs.

\section{Abundance of differentially expressed TF unigenes in response to salt stress in tolerant and sensitive genotypes}

To govern necessary transcriptional regulation in response to developmental and environmental conditions, plant genomes encode a large number of TFs. In the present study, we identified 3,202 TF unigenes (see Additional file 2A) by global TF classification of DEUs. Many TFs in the AP2/EREBP family, followed to a lesser extent by WRKY, MYB, NAC, DBP, bHLH, C2H2, C3H, C2C2, and CCAAT family members, were up-regulated during salt stress (Figure 2 and Additional file 2B). Some members of these families have been reported to participate in abiotic stress response, suggesting their roles in plant adaptation to stress [18,59-62]. Seven unigene clusters were significantly assigned as comprising co-expressed genes according to STEM (Figure 3A). The mechanism responsible for these gene co-expression clusters is unclear, although the co-expression of clustered genes may be partially regulated by the interaction of common elements and TFs [63]. A previous promoter analysis has suggested that some of these co-expressed genes may be regulated by common TFs [64]. In light of the above views, we separated out the differentially co-expressed unigenes from all differentially expressed genes encoding TFs to identify the types of TFs regulating response to 4and 24-h salt stress (Table 5 and Additional file $2 \mathrm{C}-\mathrm{H}$ ).

\section{Salt-responsive TF unigenes in the two cotton genotypes}

Dramatic changes were observed in TF unigene abundance and types (common and specific) at 4- and 24-h salt-stress time points in the two contrasting genotypes. Among these changes, $478 \mathrm{TF}$ unigenes (groups III and IV in Table 6) exhibited similar patterns of altered expression between the two genotypes. This dramatic variation is consistent with the plant switching from normal growth and development to stress-specific responses. Our observation is the first reported up-regulation of 
GhbZIP, GhDREB1C, GhMYB, GhMYB2, and GhWAKY under 4- and 24-h salt-stress treatment. AtRAP2-12 and AtRAP2-3, homologs of cotton genes GhERT and GbERF2, are reported to increase rapidly in Arabidopsis under salt stress $[65,66]$. Our results illuminate the roles of these genes in response to salt stress (in addition to abiotic stresses). In an earlier study, bHLH family member GhbHLH1 was transitorily induced in leaves by abscisic acid (ABA) and polyethylene glycol treatments [67]. In our study, GhbHLH1 was significantly up-regulated (see Additional file 2E), indicating its additional involvement in cotton salt-stress response. Seven TF genes (three GhNAC, two GhHB and two GhMYB genes) were identified at 4- and 24-h salt-stress time points, suggesting that their protein products may play an important role in salt-stress response in addition to the involvement in ABA signaling uncovered in previous research $[22,68,69]$. Transcripts encoding C2H2-type zinc finger proteins (ZAT10) that have been implicated in salt stress tolerance were up-regulated under 4- and 24-h salt stress. Overexpression of C2H2-type zinc finger proteins (STZ/ZAT1O homologs) has been shown to induce the expression of several stress genes related to salt tolerance, dehydration, and cold stress [70,71]. GhMADS7 exhibited higher expression levels in the two cotton genotypes upon 4 and $24 \mathrm{~h}$ of salt treatment. Although GhMADS7 is a wellknown participant in cotton fiber elongation, salt-stress response is a novel function for this TF; alternative splicing may have altered its cellular role [72].

Although 115 "osmotic stress"-specific TF unigenes were observed after $4 \mathrm{~h}$ of stress treatment, most of the 226 "salt stress"-specific TF unigenes appeared to be ion-specific at $24 \mathrm{~h}$ (groups I, II, IV, and IV in Table 5). These two salt-stress treatment phases clearly triggered very different responses in the two contrasting genotypes. Another finding of our study was that 37 unigenes belonging to the AP2/EREBP family of TFs were also related to salt tolerance. DREB/CBF subfamily genes, such as DREB2A [73,74] and DREB2B [75], are reportedly involved in osmotic $(4 \mathrm{~h})$ and ionic $(24 \mathrm{~h})$ stress. Interestingly, we also found that some type-B ARRs (e.g., AtPRR7) were induced or repressed at 4- and 24-h time points (Additional file 2C, D). These general salt-responsive genes identified at $4 \mathrm{~h}$ (osmotic stress) are thus similar to those identified at $24 \mathrm{~h}$ (ionic stress). Because those unigenes were expressed in both tolerant and sensitive genotypes, they may not be directly responsible for salt tolerance.

\section{Salt-responsive TF unigenes in the salt-tolerant cotton genotype}

We detected 129 unigenes-48 at 4 h, 42 at 24 h, and 39 at both time points (Table 5 and Additional file $2 \mathrm{~F}$, $\mathrm{G}, \mathrm{H})$ - that were differentially expressed under $\mathrm{NaCl}$ treatment in the salt-tolerant genotype but not in the salt-sensitive one. In addition, unigene expression in the tolerant line Earlistaple 7 differed dramatically between 24- vs. 4-h salt stress (Table 5). One possible explanation for this temporal variation is that the expression of upstream response genes (including TFs) was gradually reduced during osmotic stress recovery, with the upstream cascade genes then activated during ionic stress. Other putative TFs related to salt tolerance that were identified in this study included AP2-EREBP, MYB, C2C2-Dof, bZIP, and bHLH (see Additional file 2). Previous research has also demonstrated that some differentially expressed TF genes are associated with plant salt tolerance, including GhHB2 [68], GhDREB1A [76], AtC3H29 [59], AtPP2C66 [77], and OsC3H33 [78]. The discovery in this study of many TFs in various families indicates that a complicated transcriptional regulation network is involved in response to high salinity in cotton.

\section{Differentially expressed regulators and functional unigenes in tolerant and sensitive genotypes under salt stress}

When a plant experiences sudden salt stress, salinity perception occurs via ionic and osmotic stress signaling. These signals are first sensed by receptors present on plant cell membranes, with a large number of cellmembrane transport pathways, including ion channels and ion carriers, also participating in this signal transduction. Many of these receptors and transporters have been characterized at the gene level, revealing that the uptake and distribution of particular ions may involve some of the genes and gene families identified in our study (see Additional file 4 and Figure 11). Plant cyclic nucleotide-gated ion channels (CNGCs) are mainly involved in developmental processes, plant-pathogen interactions, and initiation of cytosolic $\mathrm{Ca}^{2+}$-dependent signal transduction. In our study, GhCNGC was identified and differentially expressed under 4- and 24-h salt stress in the salt-tolerant genotype. CNGC3 has only limited involvement in long-term $\mathrm{Na}^{+}$uptake, as revealed by an earlier study that found that short-term uptake of $\mathrm{Na}^{+}$was greater in the $c n g c 3$ mutant relative to the wild type, but uncovered no significant differences in $\mathrm{Na}^{+}$content after prolonged periods [79]. Several other key $\mathrm{ABC}$ transporter and $\mathrm{H}^{+}$-transporting ATPase genes, such as $A B C B 21, A B C C 10, A B C G 39, A H A 1, A H A 5$, and $V A M P 722$, were significantly up-regulated at 4 and/or $24 \mathrm{~h}$ of salt treatment. Extracellular stress signals are first perceived by membrane receptors, which then activate a large and complex intracellular signaling cascade that includes the generation of second messengers such as $\mathrm{Ca}^{2+}$. This increased cytosolic $\mathrm{Ca}^{2+}$ initiates the stress signaling pathways required for stress tolerance [80].

CDPK, SOS, and MAPK pathways are important signaling pathways associated with salt stress response [81]. 


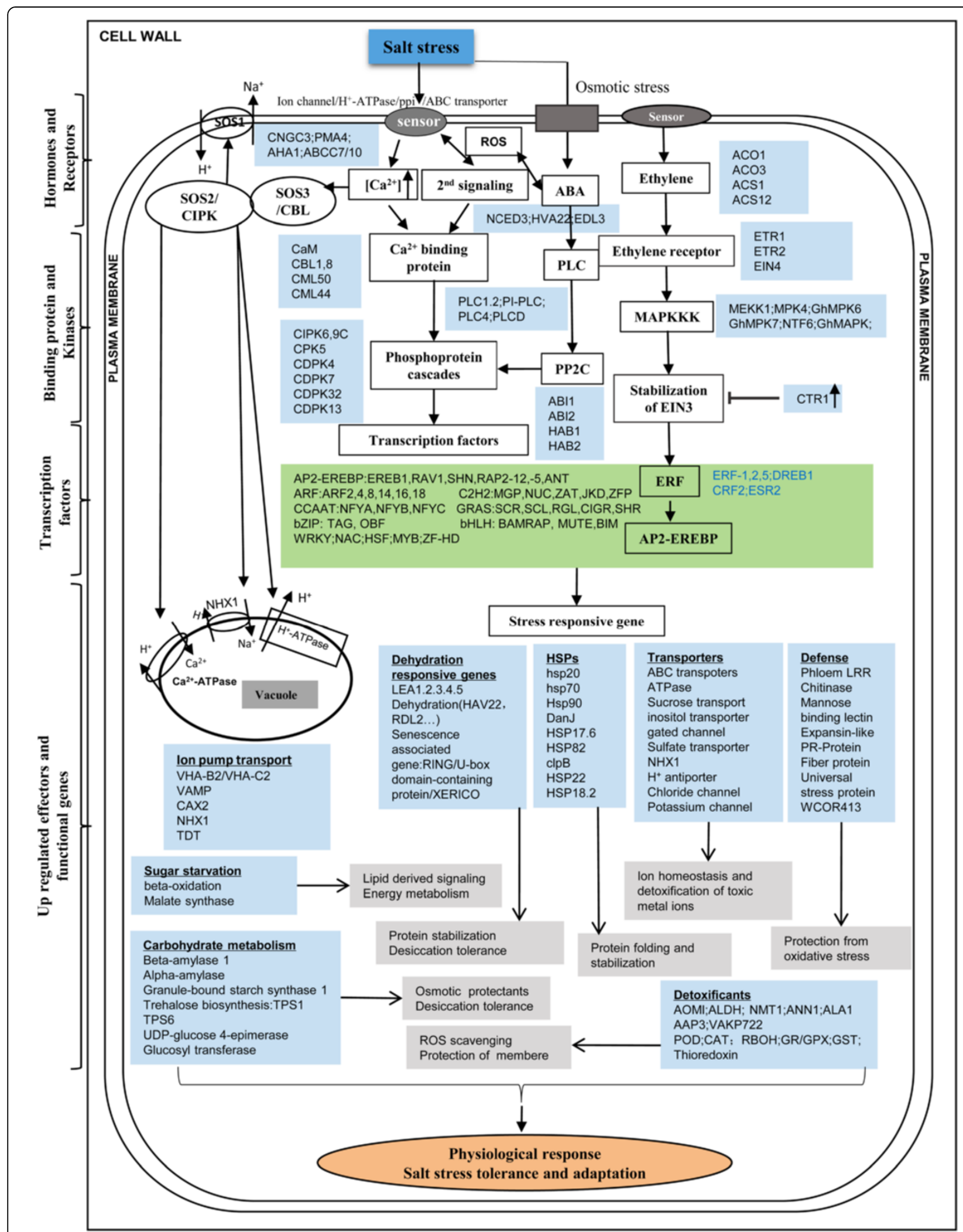

Figure 11 Overview of genes differentially expressed under salt stress at $\mathbf{4}$ and $\mathbf{2 4} \mathbf{h}$ in Gossypium hirsutum. Up-regulated genes (wathet blue and green) expressed at various stages ( 4 and $24 \mathrm{~h}$ ) are indicated in the boxes. Receptor, kinase, TF, and effector genes that were differentially expressed under salt stress are listed in Additional file 4. 
CaMs/CMLs, CDPKs, and CIPKs represent the three major classes of plant $\mathrm{Ca}^{2+}$ sensors that are involved in many developmental and stress-induced signal transduction pathways [82]. In the present study, CDPKs (homologous to $A t C P K 7$ and $A t C P K 30$ ) were mainly up-regulated at 4 and $24 \mathrm{~h}$ in the salt-tolerant genotype, with their expression remaining at low levels over $24 \mathrm{~h}$ of salt stress in the salt-sensitive genotype. We also found that a CPK5 gene, homologous to PtCPK5, was up-regulated at 4 and 24 h. GhCDPK32, homologous to AtCPK32, was identified and exhibited peak expression at $24 \mathrm{~h}$ of treatment (see Additional file 4). AtCPK32 overexpression has been reported to affect the expression of several ABF4-regulated genes and to be involved in ABA/saltstress response [83]. Furthermore, a GhCPK5 gene was cloned and found to be responsive to salt tolerance [84]. Overexpression of OsCPK7 and AtCPK30 enhances tolerance to cold and to salinity and drought $[85,86]$. The observed up-regulation of MEKK1-MPK4/6 at 4 and $24 \mathrm{~h}$ of salt treatment indicates that the MAPK signaling pathway mediates salt-stress signaling in cotton (Figure 11). In addition, GhMPK6 was up-regulated under salinity at two time points in the salt-tolerant genotype, while its expression remained unchanged in the salt-sensitive genotype. GhCAT1 was also over-expressed both at 4 and $24 \mathrm{~h}$ in the salt-tolerant genotype (see Additional file 4). The genes in the MAPK signaling pathway likely exist in cotton as well. The role of the MEKK1-MKK2-MPK4/MPK6 cascade in cold- and salt-stress signaling has been demonstrated in Arabidopsis [87]. Luo et al. [88] have suggested that GhMPK6 plays a major role in ABA-induced CAT1 expression and $\mathrm{H}_{2} \mathrm{O}_{2}$ production. Although GhMPK7 has an important function in salicylic acid (SA)-regulated broad-spectrum resistance to pathogen infection [89], it was a novel MAPK involved in salt-stress response. The expression of genes involved in MAPK and $\mathrm{Ca}^{2+}$ signaling pathways, along with other kinases, at various time points under salt stress suggests that the induction of signaling pathways can activate responsive targets for adaptation to salt stress in cotton.

Plant hormones such as ABA, ethylene (ET), SA, and jasmonate (JA) are essential for plant adaptation to abiotic stress conditions [90]. In our study, cotton unigenes associated with ABA and ET signaling pathways showed differential expression at different time points under salt stress (Figure 11 and Additional file 4). Four genes homologous to AtNCED3, AtHVA22A, AtHVA22E, and $A t E D L 3$, which are related to $\mathrm{ABA}$ synthesis or regulation, were up-regulated after 4 and $24 \mathrm{~h}$ of salt treatment in the salt-tolerant line. Up-regulation of $P L C 2, A B I 1 / 2$, and $H A B 1 / 2$ at 4 and $24 \mathrm{~h}, P L C 4$ at $4 \mathrm{~h}$, and PLC1 at $24 \mathrm{~h}$ in the salt-tolerant genotype implied that $A B A$ signaling pathway induction occurred under salt stress (Figure 11). As one of the main plant hormones, ABA regulates numerous developmental processes and adaptive stress responses in plants. Recent studies have shown that 9cisepoxycarotenoid dioxygenase (NCED3) is the key enzyme involved in ABA biosynthesis [91]. AtHVA22 is differentially regulated by $\mathrm{ABA}$, cold, dehydration, and salt stresses [92]. In A. thaliana, strong and rapid induction of the EDL3 gene has been observed under osmotic stress and high salinity [93]. Phospholipase C (PLC) and protein phosphatase 2C (PP2C) are involved in the ABA signaling pathway [94]. During salt stress (between 4 and $24 \mathrm{~h}$ ), genes related to ET biosynthesis (homologs of ACS1, ACS12, ACO1, and ACO3) were also up-regulated. ET receptor (ETR1, ETR2, and EIN4), ET signaling pathway (EIN3, ERF1, ERF2, and MEKK1-MKK2MPK4/6 kinases), and feedback mechanism (CTR1) genes were also up-regulated at 4- and/or 24-h time points (Figure 11 and Additional file 4). ET regulates stress and defense responses along with many key events of plant growth and development. It binds to membrane protein receptors (ETR1, ETR2, ERS1, ERS2, and EIN4) and activates the MAPK cascade (MKK9-MPK3/MPK6) which, in turn, stabilizes the EIN3 protein. EIN3 activates the expression of ERF1 involved in secondary transcription in the signaling pathway, while CTR1 (belonging to MAPKKK) acts as a negative regulator and degrades EIN3 [95]. In our study, only AtMPK6 was detected. We suspect that additional genes exist that were undetected. An earlier study also revealed that ET is involved in drought-stress tolerance as well as cotton fiber development [96]. Our results suggest the occurrence of multiple-hormone cross-talk in response to osmotic and salt stresses at different time points.

Some salt-responsive downstream genes, especially those involved in antioxidant system and transport functions (ion translocations across plasma and vacuolar membranes), may also provide a well-characterized clue to understanding the mechanisms responsible for salt tolerance. Under salt stress, several transporters of toxic metal ions, lipids, water, and sugar molecules were differentially expressed, such as $\mathrm{ABC}$ transporters, $\mathrm{H}^{+}$-ATPase, sucrose transport, inositol transporter, gated channel, sulfate transporter, $\mathrm{NHX1}, \mathrm{H}^{+}$ antiporter, chloride channel, and potassium channel proteins. Another aspect of salt tolerance is the regulation of ROS to protect membranes and macromolecules. In our study, several genes related to detoxification were up-regulated at various time points under salt stress. These expressed genes were homologous to $A O M I, A L D H$, NMT1, ANN1, ALA1, AAP3, VAKP722, POD, CAT, RBOH, GR/GPX, and GST [97-99].

\section{Mediation of a potential regulatory network via conserved miRNAs in the $G$. hirsutum salt-tolerant genotype}

Over the past few years, miRNAs have been reported to be prominent gene regulatory factors in plant tolerance to environmental stresses such as drought, cold, heat, 
and high salinity [100]. Much effort has been devoted to understanding their role in salt-stress responses of various plants including Zea mays [39], Populus euphratica [101], Gossypium hirsutum [40], and Thellungiella salsuginea [102].

In our study, salt-responsive miRNAs were detected in both cotton genotypes. A majority of the responsive miRNAs were either detected only in the salt-tolerant genotype or had distinct and inverse expression trends at 4- or 24-h time points between the two cotton genotypes (Figure 8B; Additional file 9A). This dichotomy suggests that common-regulated miRNAs may be part of the fundamental mechanism of salt-stress adaptation, while differentially expressed miRNAs are responsible for the distinct salt sensitivities between the two cotton genotypes (see Additional file 10). Our results are consistent with a previous hypothesis that highly expressed miRNAs are primarily responsible for control of basic cellular and developmental pathways common to most eukaryotes, whereas lower-expressed miRNAs are involved in regulation of lineage-specific pathways and functions [103].

Because miRNAs regulate the specific genes targeting mRNAs for degradation or translation inhibition, identification of the potential target unigenes of miRNAs is crucially important for understanding miRNA-mediated processes such as salt tolerance in plants. According to the PCC and linear regression analysis, the candidate negative correlation miRNA/target genes were further analyzed (Figure 7). It is noted that there is four clumps along the X-axis obviously: miRNA strongly down, slightly down, slightly up, and very strongly up (Figure 7B, E). Since some miRNAs were strongly repressed in salt stress than that in control condition,and some miRNAs were strongly inducted in salt stress compared with that in control condition, the $\log _{2}(\mathrm{E} 4 / \mathrm{E} 0)$ and $\log _{2}(\mathrm{E} 24 / \mathrm{E} 0)$ value of some miRNAs in different treatment were more than 10 or less than -10 . These miRNAs may be play an important role in response to salt stress. The identified differentially expressed miRNAs which their $\log _{2}$ (E4/E0) and $\log _{2}$ (E24/E0) value range of $-2 \sim-1$ and $1 \sim 4$ can regard as slightly down and slightly up-regulated miRNAs. Therefore, the data points are separated into four clumps along the $\mathrm{X}$-axis. There is no doubt that all these candidate negative correlation miRNA/target genes should be emphasized. Here, we found that the differentially expressed, conserved miRNA targets of salt-tolerant Earlistaple 7 generally encoded TFs, enzymes, and stress response, transport, and signal transduction proteins (Figure 9).

Many miRNA target genes encode mRNAs of TFs, indicating upstream regulation of miRNAs during developmental processes and environmental responses [104,105]. Some miRNAs, including miR156, miR164, miR166, miR171, miR172, miR393, miR396, and miR397, exhibited altered expression profiles in at least one genotype in our study. Based on transcriptome data prediction results, they regulate the expression of TFs (Figure 9). In this study, three miR156s (bna-miR156a, osa-miR156f-3p, and gma-miR156q) and one miR172 (zma-miR172a) were upregulated and down-regulated, respectively, under 24-h salt treatment in the tolerant line Earlistaple 7. In addition, three of the above miRNAs may function by changing normal levels of downstream gene transcripts through modulation of SPL5 and RAP2-7 expression (see Additional file 11). miR156 and miR172 are also involved in the regulation of flowering time and floral development in Arabidopsis, which they accomplish by negatively regulating SBP-LIKE proteins (SPLs) and AP2-like factors, respectively [106]. These results suggest that the three conserved miRNAs may play significant regulatory roles in shoot maturation and the vegetative- to reproductive-phase transition. The NAC family is involved in embryo and shoot meristem development, lateral root formation, auxin signaling, and defense and abiotic stress responses [107]. The conserved miRNA ptc-miR164f, which was verified by stem-loop RT-PCR to target the NAC domain-containing protein (NAC021/AtNAC1), was highly down-regulated at 4 and $24 \mathrm{~h}$ of salt stress in Earlistaple 7 (see Additional file 11 and Figure 10B). We observed that the CUP-SHAPED COTYLEDON 2 gene (comp81541_c0_seq1; homologous to AtNAC098), which is targeted and negatively regulated by at-miR164a, was especially down-regulated only at $24 \mathrm{~h}$ in Earlistaple 7 (see Additional file 11 and Figure 9). This result suggests that the different miRNA164s may negatively regulate different NACs during at the early stage of salt stress of salt stress. The No Apical Meristem (NAC) protein is involved in plant hormonal control and defense $[108,109]$. In Arabidopsis, AtNAC1-overexpressing lines are bigger than the wild type, with larger leaves, thicker stems, and more abundant roots [108], suggesting that NAC1 may be an early auxin-responsive gene. CUP-SHAPED COTYLEDON 2 (a NAC family member) and at-miR164A are transcribed in overlapping domains at the margins of young leaf primordia, with transcription gradually restricted to the sinus where the Arabidopsis leaf margins become serrated [110]. Most often, the regulatory pathway consisting of miR393 that targets TIR1, a negative regulator in auxin signaling, may participate in lateral root development in Arabidopsis [111]. The other target was a bHLH transcription factor, which was involved in many biological processes, but there were no phenotypic changes and functional mechanisms owing to miR393/ bHLH interaction in Arabidopsis [112]. In our study, all miR393s (ath-miR393a, osa-miR393a, csi-miR393, and osa-miR393b-5p), which were highly expressed at 4 and $24 \mathrm{~h}$ of salt stress in salt-tolerant Earlistaple 7 but were unaffected or down-regulated in salt-sensitive Nan Dan 
Ba Di Da Hua, targeted and tested as negatively related to a bHLH78 homolog unigene (comp105131_c0_seq1) by stem-loop RT-PCR (see Additional file 11 and Figure 10B). Induction of miR393 at the early stage of salt stress (4 and $24 \mathrm{~h}$ in the salt-tolerant genotype) suppressed the expression of its target AtbHLH78.

In our proposed miRNA-mediated regulatory network based on miRNA-TF interactions (Figure 9), one miRNA may regulated more than one TF gene. In addition, differentially expressed conserved miRNAs may also target non-TF genes. Comparison of target genes of conserved miRNAs illustrated in the regulatory network suggests that the same miRNA response to salt stress at different time points may regulate the same or different genes to exert different functions. Nevertheless, these putative interactions need to be confirmed by experimental data.

\section{Conclusions}

In this study, we first generated a global transcription map of genes and miRNAs expressed in leaves of salttolerant cotton under salt stress. We detected 29,136 and 33,492 unigenes exhibiting differential co-expression after 4 and $24 \mathrm{~h}$ of salt stress, respectively. GO annotations demonstrated that unigenes belonging to a wide range of functional categories are involved in salinity defense response at the two different time points. We also identified 320 miRNAs conserved across the two genotypes. Of these, 108 members of miR156, miR159, miR164, miR167, miR171, miR172, miR393, miR396, miR397, and miR482 families were differentially expressed at 4 and $24 \mathrm{~h}$ under salt stress in the salt-tolerant genotype. Some miRNA targets were predicted to encode abiotic stress-responsive TFs and enzymes. Expressions of some selected differentially regulated miRNAs and their targets were found to be negatively regulated. Comparative transcriptome analysis of salt-tolerant and salt-sensitive genotypes indicated that essential salt tolerance-related genes and miRNAs were differentially expressed and were only weakly or non-responsive to salt stress in the salt-sensitive cotton. Finally, we have provided a list of salt tolerance-related genes that will facilitate candidate gene discovery and molecular marker development for salt-tolerant breeding in cotton.

\section{Methods}

\section{Plant materials and $\mathrm{NaCl}$ treatments}

Plant materials comprised two upland cotton (G. hirsutum) genotypes: salt-tolerant Earlistaple 7, which was selected from Earlistaple 808 and introduced by the AR-SEAUSDA Pee Dee Experiment Station in the United States in 1982, and salt-sensitive Nan Dan Ba Di Da Hua, introduced from Guangxi Province, China, before 1978. Seeds of the two cotton genotypes were available in the National Mid-term Genebank of the Institute of Cotton Research,
Chinese Academy of Agricultural Sciences (ICR-CAAS) after signing the Material Transfer Agreement (MTA) [113]. Seedling cultivation and all experiments were carried out at the State Key Laboratory of Cotton Biology, Anyang, China.

Hand-selected seeds (120 of each genotype) of Nan Dan Ba Di Da Hua and Earlistaple 7 were surface-sterilized in $70 \%(\mathrm{v} / \mathrm{v})$ ethanol for $15 \mathrm{~s}$ and $4 \%(\mathrm{w} / \mathrm{v})$ sodium hypochlorite for $15 \mathrm{~min}$, and then rinsed several times with sterile distilled water. The seeds were submerged in sterile water for $12 \mathrm{~h}$ at room temperature and then sown in sterile silica sand. After 3 days, germinated seedlings were transferred to hydroponic containers $(600 \times 350 \times 120 \mathrm{~mm})$ containing half-strength Hoagland's solution (pH 6.0) [114] and grown in a phytotron (KR-III; Zhengzhou Henan Ke-rui Inc.). Growth conditions were 28/22 day/night temperature, $60-80 \%$ relative humidity, and a $14 \mathrm{~h} / 10 \mathrm{~h}$ light/dark cycle under $450 \mu \mathrm{mol} \cdot \mathrm{m}^{-2}$.s light intensity. The culture solutions were changed after about 14 days, when seedlings had produced three leaves. Seedlings showing normal growth were randomly divided into two groups; one group was placed into tanks filled with a $200 \mathrm{mM}$ solution of $\mathrm{NaCl}$, and the remaining seedlings were transferred to tanks filled with plain water to serve as control. After exposure to the two solutions for 4, 24, 48 , and $72 \mathrm{~h}$, seedlings of control and treated Nan Dan Ba Di Da Hua and Earlistaple 7 were harvested directly into liquid nitrogen and stored at $-80^{\circ} \mathrm{C}$ until used for RNA extraction.

\section{Leaf RWC, REC, chlorophyll content, and ion measurements}

Plant fresh weight (FW) was measured immediately after harvest. The leaves were floated on deionized water for $8 \mathrm{~h}$ at $4^{\circ} \mathrm{C}$. The turgid leaves were quickly weighed (TW) and their dry mass (DW) was measured after oven-drying at $105^{\circ} \mathrm{C}$ for $10 \mathrm{~min}$ followed by $80^{\circ} \mathrm{C}$ for $24 \mathrm{~h}$. RWC was calculated as follows: RWC $(\%)=(\mathrm{FW}-\mathrm{DW}) /(\mathrm{TW}-$ $\mathrm{DW}) \times 100$ [115]. For REC, $0.5 \mathrm{~g}$ of fresh leaves were rinsed 3 times with $\mathrm{ddH}_{2} \mathrm{O}$ and were placed in an Erlenmeyer flask containing $40 \mathrm{~mL}$ of $\mathrm{ddH}_{2} \mathrm{O}$ incubated at room temperature for $4 \mathrm{~h}$. The electrical conductivity of the solution (C1) was measured using an EM38 conductivity meter (ICT international, Armidale, NSW, Australia). The solution was boiled for $10 \mathrm{~min}$ and cooled to room temperature, and electrical conductivity (C2) was remeasured. REC was calculated as C1 / C2 × 100\% [116]. To measure leaf chlorophyll, $0.2 \mathrm{~g}$ of seedling leaves were incubated in $80 \%$ acetone in darkness at $4^{\circ} \mathrm{C}$ overnight. After centrifugation at $5000 \times g$ for $5 \mathrm{~min}$ at $4^{\circ} \mathrm{C}$, the absorbance of the supernatant was measured at 663 and $645 \mathrm{~nm}$ with a DU800 spectrophotometer (Beckman Coulter, Brea, CA, USA). Chlorophyll content was calculated as described by Porra (1989) [117]. Concentrations 
of $\mathrm{Na}^{+}$and $\mathrm{K}^{+}$in roots and leaves were determined by inductively coupled plasma-optical emission spectrometry (ICP-OES) (Optima 2100 DV; Perkin-Elmer, Wellesley USA) according to the manufacturer's instructions. Fifteen shoots per genotype were analyzed (3 from control and 12 from salt-treated plants). To ensure more accurate measurements, five independent plants were pooled as a sample (about 0.2-0.6 g) to reduce the effects of biological variation. For the $\mathrm{Na}^{+}$and $\mathrm{K}^{+}$determinations, the samples were completely digested in $12 \mathrm{~mL} 65 \% \mathrm{HNO}_{3}$ and then analyzed on the ICP-OES spectrometer against National Institute of Standards and Technology traceable standards and controls. For the physiological experiments, three or more independent biological replicates of each control and time-treated sample were performed.

\section{RNA extraction and qRT-PCR}

Total RNA was isolated from cotton leaf samples treated with either $200 \mathrm{mM} \mathrm{NaCl}$ or water (as a control) for 4 or $24 \mathrm{~h}$ using a modified CTAB method and purified using Qiagen RNeasy columns (Qiagen, Hilden, Germany). First-strand cDNA was synthesized using a PrimeScript RT reagent kit with gDNA eraser (Takara, Dalian, China) according to the manufacturer's instructions. Primers are listed in Additional file 5A. qRT-PCR was performed on a 7500 Fast Real-Time PCR system (Applied Biosystems, Inc., California USA). The $20-\mu \mathrm{L}$ reaction solutions contained $10 \mu \mathrm{L}$ SYBR Green Premix Ex Taq (Takara), $1 \mathrm{ng}$ cDNA sample, and $0.2 \mu \mathrm{M}$ gene-specific primers. Three replicate PCRs were run per cDNA sample, and three cDNAs from different samples were used as biological replicates. Relative expression levels were calculated by the $2^{-\Delta \Delta \mathrm{T}}$ method [118] using GhActin (GI: AY305733) as an internal standard to normalize cDNA content [119].

The expression profiles of 10 salt-responsive mature miRNAs were assayed by stem-loop RT-PCR. Stem-loop reverse-transcription primers were designed following the method described by Chen et al. [120] and Varkonyi-Gasic et al. [121]. Their 3' ends, which were complementary to the 6-nt $3^{\prime}$ end of the miRNA, were combined with the 44-nt sequence 5'-GTCGTATCCA GTGCAGGGTCCGAGGTATTCGCACTGGATACGA

C-3' (Additional file 5B) to constitute the primer. Subsequent PCRs used a $5^{\prime}$ primer matching the $\sim 18 \mathrm{nt}$ at the $5^{\prime}$ end of the target miRNA. The 3 ' primer was a universal reverse-transcription primer. The stem-loop reverse transcription reactions were performed using a PrimeScript RT reagent kit with gDNA eraser (Takara) according to the supplier's manual. U6 snRNA was used as a reference for miRNA expression validation. Stem-loop RT primers and miRNA-specific PCR primers are listed in Additional file 5B, C. As in the qRT-PCR analysis, the $2^{-\Delta \Delta \mathrm{T}}$ method was used to calculate relative expression levels of salt-responsive mature miRNAs.

\section{Transcriptome sequencing and de novo assembly}

Poly(A) mRNA was isolated from $10 \mu \mathrm{g}$ total RNA of each leaf sample using oligo (dT) beads. The mRNA was sheared into short fragments with fragmentation buffer and selected for construction of Illumina RNA-Seq libraries according to the described protocol. Each library had an insert size of approximately $200 \mathrm{bp}$ and was sequenced on a HiSeq 2000 system (Illumina, California USA). Leafsample read lengths were $90 \mathrm{bp}$. The raw image data was transformed by base-calling into sequence data and stored in FASTQ format. To control for the influence of error rate in the Solexa data results, quality pretreatment of the raw data was performed: the sliding window method was first used to remove low-quality fragments (quality threshold $=20$, error rate $=1 \%$, window size $=5 \mathrm{bp}$, and length threshold $=35 \mathrm{bp})$, followed by removal of partial $(<35 \mathrm{bp})$ sequences containing " $\mathrm{N}$ " from the reads. After filtering of low-quality and dirty raw reads, transcriptome de novo assembly was carried out using the pairedend assembly method as implemented in Trinity (http://sourceforge.net/projects/trinityrnaseq/files/; trinityrnaseq_r2012-10-05) [122]. After removal of repeats from the spliced sequences, transcripts were obtained of the expected length and size. The longest transcript in each locus ("comp * _ " c"; Chrysalis Clusters module) was taken as the unigene. To assign possible annotations, all unigene sequences were searched using Blastx $\left(e\right.$-value $\left.<10^{-5}\right)$ against the following protein databases: NR (in NCBI), SWISSPROT (UniProt), TrEMBL (http://www.bioinfo.pte.hu/ more/TrEMBL.htm), CDD (in NCBI http://www.ncbi. nlm.nih.gov/cdd/), PFAM (http://pfam.janelia.org/), and KOG (http://www.ncbi.nlm.nih.gov/COG/). GO functional annotations were obtained using the Blast2GO program [123], and all unigenes were assigned into GO functional categories of molecular function, biological process, and cellular components using WEGO software [124]. To identify possible TFs in the transcripts, we used the BLAST tool, sequence information in the PlantTFDB database (http://planttfdb.cbi.pku.edu.cn), and annotations from the SWISS-PROT and NR databases.

\section{Identification and functional analysis of DEUs}

To identify genes displaying significant expression changes during $\mathrm{NaCl}$ treatment, differentially expressed transcripts were analyzed by comparing 4- and 24-h libraries with the control library, with the number of reads in all six libraries normalized to reads per kilobase of exon model per million mapped reads (RPKM) [125]. For better accuracy, only unigenes longer than $200 \mathrm{bp}$ were included in the differential unigene expression analysis. Using Bowtie v0.12.8 software and the single-end mapping method, each read per unigene was counted as $1 / \mathrm{n}$ by permitting alignment of one read on multiple unigenes. RPKM were calculated 
according to the formula RPKM $=10^{6} \times \mathrm{C} / 10^{-3} \times \mathrm{N} \times \mathrm{L}$, where $C$ is the number of reads uniquely aligned to the unigene, $\mathrm{N}$ is the total number of reads uniquely aligned to all unigenes in the specific sample, and $\mathrm{L}$ is the number of $n t$ in the unigene. The $P$-value corresponding to a differentially expressed unigene in two samples was determined from Audic's algorithm [126], with the FDR method applied to determine the $P$-value threshold in multiple tests. To determine whether a unigene was differentially expressed between two samples, we therefore used threshold criteria based on the FDR statistical method and the ratio of RPKMs, i.e., FDR $<0.001$ and $\mid \log _{2}$ Ratio $\mid \geq 1$.

Gene expression patterns uncovered during the investigated two time points were analyzed and interpreted using STEM v1.3.8 [127]. GO enrichment analyses for differentially co-expressed unigenes were performed with the differential and full unigene sets treated as prospect and background, respectively, using the hypergeometric distribution algorithm "phyper" in STEM. $P$-values $(<0.05$ or 0.01 ) were calculated between the prospective unigene and selected GO subcategories, followed by FDR adjustment.

\section{Small RNA sequencing and identification of conserved miRNAs}

For small RNA library construction, total RNA was separately extracted from the leaves of the same six samples used for generation of mRNA-seq libraries. After confirming RNA quality by agarose gel electrophoresis (28S:18S > 1.5) and on an Agilent 2100 bioanalyzer (RNA Integrity Number $\geq 8.0$ ), the extracted RNA was separated by $15 \%$ denaturing polyacrylamide gel electrophoresis (PAGE) to recover the population of small RNAs (size range 17-35 nt) present. The small RNAs were ligated at their $5^{\prime}$ and $3^{\prime}$ ends to a pair of Solexa adapters, and the resulting ligation products were gelpurified by $10 \%$ denaturing PAGE and reverse transcribed. The cDNAs obtained in this fashion were sequenced on an Illumina HiSeq 2000 system (Illumina, California USA). After removal of low-quality reads and adapter sequences from the Solexa sequencing reads, unique sequences of 17-35 nt were used for further analysis. First, the unique sequences were queried against ribosomal and transfer RNAs from tRNAdb (http://trna. bioinf.uni-leipzig.de/DataOutput/Search), SILVArRNA (http://www.arb-silva.de/), and NONCODE v3.0 (http:// www.noncode.org/NONCODERv3/; plant database only, single-nt mismatches allowed) databases to obtain matching rRNA, tRNA, snRNA, and snoRNA sequences. Any small RNA sequences having exact matches to these sequences were removed from the analysis. The remaining unique small RNA sequences were then BLASTn-searched against the conserved plant miRNAs in the miRBase database (miRBase 19.0; www.mirbase.org/) to identify conserved miRNAs. Only perfectly matched sequences were considered to be conserved miRNAs. Because we were only conducting a combined analysis of transcriptome and conserved miRNA data, we did not perform a novel miRNA prediction on the content.

\section{Analyses of conserved miRNA differential expression, target unigene prediction, and miRNA-target pair functional regulatory relationships}

A high-throughput sequencing abundance profile analysis was performed based on the sequence reads in each library. miRNA expression abundance in each library was calculated as RPM (reads per million) according to the formula $\mathrm{RPM}=$ mapped reads $\times 10^{6} /$ total reads. Calculation of $P$-values for comparing the miRNA expression between salt-treated samples and the control sample was based on previously established methods [126,128]. Specifically, we used the following $\log _{2}$ Ratio formula: $\log _{2}$ Ratio $=\log _{2}$ (miRNA reads in the salt treatment / miRNA reads in the control). Differential expression of miRNAs in the comparisons N4/N0 (where N is Nan Dan Ba Di Da Hua and $\mathrm{E}$ is Earlistaple 7), N24/N0, E4/E0, and E24/E0 were screened based on the chi-square test with filters of $P<0.05$ and $\mid \log _{2}$ Ratio $\mid \geq 1.0$.

To search target genes, the conserved miRNA sequences were matched against the cotton transcriptome unigene database using psRNATarget (http://plantgrn.noble.org/ psRNATarget/). Sequences with less than 3 nt mismatches compared with the query miRNA sequences were selected.

\section{Statistical analysis}

Each data point represented the mean of three or more replicated treatments, with each treatment consisting of at least five plants. Physiological data statistical analyses were performed by Tukey's method of two-way ANOVA using IBM SPSS Statistics v19.0 (SPSS Inc., Chicago, IL, USA). $P$-values less than 0.05 were considered as statistically significant. R software (www.r-project.org) was used for the calculation of Pearson correlation coefficients between miRNAs and their targets and for the construction of a heatmap of co-expressed TF unigenes and miRNA expression profiles.

\section{Additional files}

\footnotetext{
Additional file 1: Summary of de novo assembled mRNA-seq transcriptome data. (A) Mapping of the length distribution of all unigenes; (B) GC content of all unigenes; (C) Functional classifications of GO-annotated unigenes.

Additional file 2: The characteristic of salt-responsive transcription factor (TF) unigenes in different comparisons. Eight charts are included. (A) Annotation of 3,172 TF unigenes from all samples; (B) Numbers of differentially expressed unigenes (up and down-regulation) in each transcription factor family for four comparisons; (C) TF unigenes
} 
commonly differentially expressed in N-E only at $4 \mathrm{~h}$; (D) TF unigenes commonly differentially expressed in N-E only at $24 \mathrm{~h}$; (E) TF unigenes commonly differentially expressed in N-E both at 4 and $24 \mathrm{~h}$; (F) TF unigenes differentially expressed only in $E$ at $4 \mathrm{~h}$; (G) TF unigenes differentially expressed only in E at 24 h; (H) TF unigenes differentially expressed only in E both at 4 and $24 \mathrm{~h}$.

Additional file 3: Flowchart analysis of all differentially co-expressed unigenes.

Additional file 4: Differentially expressed unigenes at 4- and 24-h of salt stress in Earlistaple 7 (also shown in Figure 11).

Additional file 5: Specific primer information of unigenes (including 9 miRNA targets) for qRT-PCR and 9 conserved miRNAs for stem-loop RT-PCR. (A) 26 pairs of transcript-specific primers for qRT-PCR; (B) 9 miRNA primers for stem-loop RT-PCR; (C) 9 miRNA gene primers for real time PCR analysis; (D) 26 represented unigenes QRT-PCR data and mRNA-seq data in four comparisons.

Additional file 6: Summary of small RNA sequencing data. (A) Statistical summary of data before and after quality pretreatment. (B) Size distribution of small RNA unique reads; (C) Number of detected members of each miRNA family; (D) Abundance of conserved miRNA families.

Additional file 7: RPM values of (A) all identified and (B) Gossypium hirsutum conserved miRNAs in six libraries.

Additional file 8: Differentially expressed conserved miRNAs at 4- and 24-h of salt stress in Nan Dan Ba Di Da Hua and Earlistaple 7.

Additional file 9: Venn diagram illustrating the numbers of differently up- and down-regulated miRNAs between Nan Dan Ba Di Da Hua and Earlistaple 7 in response to salt stress at (A) $4 \mathrm{~h}$ and (B) $24 \mathrm{~h}$; (C) Flowchart analysis of differentially expressed miRNAs and combined analysis with their target genes; (D) Procedure for screening of negative miRNA-target unigene relationships related to salt-treatment response; $(\mathrm{E})$ Gene Ontology analysis of conserved miRNA targets identified in salt-tolerant Earlistaple 7 at 4 and $24 \mathrm{~h}$ of salt treatment.

Additional file 10: Detailed information for potential targets of conserved miRNAs at 4- and 24-h of salt stress in Earlistaple 7. (A) Negatively correlated miRNA-target pairs in Earlistaple 7 at 4-hi

(B) Positively correlated miRNA-target pairs in Earlistaple 7 at 4-h;

(C) Negatively correlated miRNA-target pairs in Earlistaple 7 at 24-h

(D) Positively correlated miRNA-target pairs in Earlistaple 7 at 24-h.

Additional file 11: Detailed information on differentially expressed conserved miRNAs in different comparisons. (A) RPM values of 108 differentially expressed conserved miRNAs at 4- or 24-h time points in five comparisons; (B) The genotype-specific salt-responsive conserved miRNAs at 4- or 24-h time points of salt-stress in five comparisons; (C) Seventy-five negatively correlated miRNA-target pairs in salt-stressed Earlistaple 7 at 4- and 24-h time points; (D) Matching of 75 negatively correlated miRNA-target pairs; (E) The salt-responsive differentially expressed conserved miRNAs and their target genes at different treatment time points (relationship shown in Figure 9).

\section{Abbreviations}

N: Nan Dan Ba Di Da Hua; E: Earlistaple 7; RWC: Relative water content; REC: Relative electric conductivity; GO: Gene ontology; DEU: Differentially expressed unigene; FDR: False discovery rate; RPKM: Reads per kilobase of gene model per million mapped reads; RPM: Reads per million; qRT-PCR: Real-time quantitative RT-PCR; SLRT-PCR: Stem-loop reverse transcription-PCR; STEM: Short time-servies expression miner;

TF: Transcription factor; PCC: Pearson correlation coefficient.

\section{Competing interests}

The authors declare that they have no competing interests.

\section{Authors' contributions}

$X M D$ received grant support. $X M D$ and $Y L L$ designed the experiment. ZP and FFX grew cotton seedlings, performed the salt stress treatments, collected the tissue samples, and physiological experiments. JLS, ZEP participated in the preliminary data analysis. SPH, WFG, YLL and XMD revised the manuscript. ZP analyzed the physiological and transcriptome results, validated gene and miRNA expression, and prepared the manuscript. All authors reviewed and approved the final manuscript.

\section{Acknowledgements}

This work was supported by the grant of the "Twelfth Five-Year Plan" National Science and Technology Support Program of China [No. 2013BAD01B03]

Received: 30 April 2014 Accepted: 4 August 2014

Published: 5 September 2014

\section{References}

1. Wang X, Fan P, Song H, Chen X, Li X, Li Y: Comparative proteomic analysis of differentially expressed proteins in shoots of Salicornia europaea under different salinity. J Proteome Res 2009, 8:3331-3345.

2. Rozema J, Flowers T: Crops for a salinized world. Science 2008, 322:1478-1480.

3. Zhao KF, Fan H, Song J: Species, types, vegetation of halophytes in China and its economic potential. In Utilization of halophytes and sustainable development of local agriculture. Beijing: China Meteorological Press; 2002:1-9.

4. Hasegawa PM, Bressan RA, Zhu J-K, Bohnert HJ: Plant cellular and molecular responses to high salinity. Annu Rev Plant Physiol Plant Mol Biol 2000, 51:463-499.

5. Munns R: Comparative physiology of salt and water stress. Plant Cell Environ 2002, 25:239-250.

6. Munns R: Genes and salt tolerance: bringing them together. New Phytol 2005, 167:645-663.

7. Loescher W, Chan Z, Grumet R: Options for Developing Salt-tolerant Crops. HortScience 2011, 46:1085-1092.

8. Maas E: Salt tolerance of plants. Handb Plant Sci Agric 1984, 2:57-75.

9. Leidi $E O$, Saiz J: Is salinity tolerance related to $\mathrm{Na}$ accumulation in upland cotton (Gossypium hirsutum) seedlings? Plant and Soil 1997, 190:67-75.

10. Ahmad S, Khan N, lqbal M, Hussain A, Hassan M: Salt tolerance of cotton (Gossypium hirsutum L.). Asian J Plant Sci 2002, 1:715-719.

11. Reinhardt $D$, Rost T: Primary and lateral root development of dark-and light-grown cotton seedlings under salinity stress. Botanica Acta (Germany) 1995, 108:457-465

12. Leidi E: Genotypic variation of cotton in response to stress by $\mathrm{NaCl}$ or PEG. REUR Technical Series 1994

13. Brugnoli E, Lauteri M: Effects of salinity on stomatal conductance, photosynthetic capacity, and carbon isotope discrimination of salt-tolerant (Gossypium hirsutum L.) and salt-sensitive (Phaseolus vulgaris L.) C3 non-halophytes. Plant Physiol 1991, 95:628-635.

14. Flowers T: Improving crop salt tolerance. J Exp Biol 2004, 55:307-319.

15. Chinnusamy V, Zhu J, Zhu J-K: Salt stress signaling and mechanisms of plant salt tolerance. In Genetic engineering: Springer; 2006:141-177.

16. Roy SJ, Tucker EJ, Tester M: Genetic analysis of abiotic stress tolerance in crops. Curr Opin Plant Biol 2011, 14:232-239.

17. Wu C-A, Yang G-D, Meng Q-W, Zheng C-C: The cotton GhNHX1 gene encoding a novel putative tonoplast $\mathrm{Na}^{+} / \mathrm{H}^{+}$antiporter plays an important role in salt stress. Plant Cell Physiol 2004, 45:600-607.

18. Gao S-Q, Chen M, Xia L-Q, Xiu H-J, Xu Z-S, Li L-C, Zhao C-P, Cheng X-G, Ma $Y$-Z: A cotton (Gossypium hirsutum) DRE-binding transcription factor gene, GhDREB, confers enhanced tolerance to drought, high salt, and freezing stresses in transgenic wheat. Plant Cell Rep 2009, 28:301-311.

19. Jin LG, Li H, Liu JY: Molecular characterization of three ethylene responsive element binding factor genes from cotton. J Integr Plant Biol 2010, 52:485-495.

20. Jin L-G, Liu J-Y: Molecular cloning, expression profile and promoter analysis of a novel ethylene responsive transcription factor gene GhERF4 from cotton (Gossypium hirsutum). Plant Physiol Biochem 2008, 46:46-53.

21. Champion A, Hébrard E, Parra B, Bournaud C, Marmey P, Tranchant C, Nicole M: Molecular diversity and gene expression of cotton ERF transcription factors reveal that group IXa members are responsive to jasmonate, ethylene and Xanthomonas. Mol Plant Pathol 2009, 10:471-485.

22. Meng C, Cai C, Zhang T, Guo W: Characterization of six novel NAC genes and their responses to abiotic stresses in Gossypium hirsutum L. Plant Sci 2009, 176:352-359.

23. Xue T, Li X, Zhu W, Wu C, Yang G, Zheng C: Cotton metallothionein GhMT3a, a reactive oxygen species scavenger, increased tolerance against abiotic stress in transgenic tobacco and yeast. J Exp Biol 2009, 60:339-349. 
24. Zhang L, Xi D, Li S, Gao Z, Zhao S, Shi J, Wu C, Guo X: A cotton group C MAP kinase gene, GhMPK2, positively regulates salt and drought tolerance in tobacco. Plant Mol Biol 2011, 77:17-31.

25. Lu W, Chu X, Li Y, Wang C, Guo X: Cotton GhMKK1 Induces the Tolerance of Salt and Drought Stress, and Mediates Defence Responses to Pathogen Infection in Transgenic Nicotiana benthamiana. PLoS One 2013, 8:e68503.

26. Guo YH, Yu YP, Wang D, Wu CA, Yang GD, Huang JG, Zheng CC: GhZFP1, a novel $\mathrm{CCCH}$-type zinc finger protein from cotton, enhances salt stress tolerance and fungal disease resistance in transgenic tobacco by interacting with GZIRD21A and GZIPR5. New Phytol 2009, 183:62-75.

27. Kawasaki S, Borchert C, Deyholos M, Wang H, Brazille S, Kawai K, Galbraith D, Bohnert HJ: Gene expression profiles during the initial phase of salt stress in rice. Plant Cell 2001, 13:889-905.

28. Dai Yin C, LUO YH, Min S, Da L, LIN HX: Salt-responsive genes in rice revealed by cDNA microarray analysis. Cell Res 2005, 15:796-810

29. Ueda A, Kathiresan A, Bennett J, Takabe T: Comparative transcriptome analyses of barley and rice under salt stress. Theor Appl Genet 2006, 112:1286-1294.

30. Rodriguez-Uribe L, Higbie SM, Stewart JM, Wilkins T, Lindemann W, Sengupta-Gopalan C, Zhang J: Identification of salt responsive genes using comparative microarray analysis in Upland cotton (Gossypium hirsutum L.). Plant Sci 2011, 180:461-469.

31. Yao D, Zhang X, Zhao X, Liu C, Wang C, Zhang Z, Zhang C, Wei Q, Wang Q, Yan $\mathrm{H}$ : Transcriptome analysis reveals salt-stress-regulated biological processes and key pathways in roots of cotton (Gossypium hirsutum L). Genomics 2011, 98:47-55.

32. Wang G, Zhu Q, Meng Q, Wu C: Transcript profiling during salt stress of young cotton (Gossypium hirsutum) seedlings via Solexa sequencing. Acta Physiol Plant 2012, 34:107-115.

33. Xu P, Liu Z, Fan X, Gao J, Zhang X, Zhang X, Shen X: De novo transcriptome sequencing and comparative analysis of differentially expressed genes in Gossypium aridum under salt stress. Gene 2013, 525:26-34.

34. Carrington JC, Ambros V: Role of microRNAs in plant and animal development. Science 2003, 301:336-338.

35. Schwab R, Palatnik JF, Riester M, Schommer C, Schmid M, Weigel D: Specific effects of microRNAs on the plant transcriptome. Dev Cell 2005, 8:517-527.

36. Brodersen $P$, Sakvarelidze-Achard L, Bruun-Rasmussen M, Dunoyer P, Yamamoto $Y$, Sieburth $L$, Voinnet $O$ : Widespread translational inhibition by plant miRNAs and siRNAs. Science 2008, 320:1185-1190.

37. Liu H-H, Tian X, Li Y-J, Wu C-A, Zheng C-C: Microarray-based analysis of stress-regulated microRNAs in Arabidopsis thaliana. RNA 2008, 14:836-843.

38. Sunkar R, Zhou X, Zheng Y, Zhang W, Zhu J-K: Identification of novel and candidate miRNAs in rice by high throughput sequencing. BMC Plant Bio 2008, 8(1):25.

39. Ding D, Zhang L, Wang H, Liu Z, Zhang Z, Zheng Y: Differential expression of miRNAs in response to salt stress in maize roots. Ann Bot 2009, 103:29-38.

40. Yin Z, Li Y, YU J, LiU Y, Li C, Han X, Shen F: Difference in miRNA expression profiles between two cotton cultivars with distinct salt sensitivity. Mol Biol Rep 2012, 39:4961-4970.

41. Zhu J, Li W, Yang W, Qi L, Han S: Identification of microRNAs in Caragana intermedia by high-throughput sequencing and expression analysis of 12 microRNAs and their targets under salt stress. Plant Cell Rep 2013, 32:1339-1349.

42. Zhang B, Wang Q, Wang K, Pan X, Liu F, Guo T, Cobb GP, Anderson TA: Identification of cotton microRNAs and their targets. Gene 2007, 397:26-37.

43. Ruan M-B, Zhao Y-T, Meng Z-H, Wang X-J, Yang W-C: Conserved miRNA analysis in Gossypium hirsutum through small RNA sequencing. Genomics 2009, 94:263-268.

44. Pang M, Woodward AW, Agarwal V, Guan X, Ha M, Ramachandran V, Chen X, Triplett BA, Stelly DM, Chen ZJ: Genome-wide analysis reveals rapid and dynamic changes in miRNA and siRNA sequence and expression during ovule and fiber development in allotetraploid cotton (Gossypium hirsutum L). Genome Biol 2009, 10:R122.

45. Xue W, Wang Z, Du M, Liu Y, Liu J-Y: Genome-wide analysis of small RNAs reveals eight fiber elongation-related and 257 novel microRNAs in elongating cotton fiber cells. BMC Genomics 2013, 14:629.
46. Zhang X, Yao D, Wang Q, Xu W, Wei Q, Wang C, Liu C, Zhang C, Yan H, Ling Y: mRNA-seq Analysis of the Gossypium arboreum transcriptome reveals tissue selective signaling in response to water stress during seedling stage. PLoS One 2013, 8:e54762.

47. Brinker $M$, Brosché $M$, Vinocur B, Abo-Ogiala A, Fayyaz $P$, Janz D, Ottow EA, Cullmann AD, Saborowski J, Kangasjärvi J: Linking the salt transcriptome with physiological responses of a salt-resistant Populus species as a strategy to identify genes important for stress acclimation. Plant Physiol 2010, 154:1697-1709.

48. Zhang X, Zhen J, Li Z, Kang D, Yang Y, Kong J, Hua J: Expression profile of early responsive genes under salt stress in upland cotton (Gossypium hirsutum L.). Plant Mol Biol Rep 2011, 29:626-637.

49. Z-h D, Zheng L-I, Wang J, Gao Z, Wu S-b, Qi Z, Wang Y-c: Transcriptomic profiling of the salt-stress response in the wild recretohalophyte Reaumuria trigyna. BMC Genomics 2013, 14:29.

50. Munns R, Tester M: Mechanisms of salinity tolerance. Annu Rev Plant Biol 2008, 59:651-681.

51. Hey SJ, Byrne E, Halford NG: The interface between metabolic and stress signalling. Ann Bot 2010, 105:197-203.

52. Lopez-Gomollon S, Mohorianu I, Szittya G, Moulton V, Dalmay T: Diverse correlation patterns between microRNAs and their targets during tomato fruit development indicates different modes of microRNA actions. Planta 2012, 236:1875-1887.

53. Flowers T, Hajibagherp M, Yeo A: Ion accumulation in the cell walls of rice plants growing under saline conditions: evidence for the Oertli hypothesis. Plant Cell Environ 1991, 14:319-325.

54. Zhu J-K: Regulation of ion homeostasis under salt stress. Curr Opin Plant Biol 2003, 6:441-445.

55. Walia H, Wilson C, Condamine P, Liu X, Ismail AM, Zeng L, Wanamaker SI, Mandal J, Xu J, Cui X: Comparative transcriptional profiling of two contrasting rice genotypes under salinity stress during the vegetative growth stage. Plant Physiol 2005, 139:822-835.

56. Ouyang B, Yang T, Li H, Zhang L, Zhang Y, Zhang J, Fei Z, Ye Z: Identification of early salt stress response genes in tomato root by suppression subtractive hybridization and microarray analysis. J Exp Biol 2007, 58:507-520.

57. Wang Y, Yang L, Zheng Z, Grumet R, Loescher W, Zhu J-K, Yang P, Hu Y, Chan Z: Transcriptomic and Physiological Variations of Three Arabidopsis Ecotypes in Response to Salt Stress. PLoS One 2013, 8:e69036.

58. Guo P, Baum M, Grando S, Ceccarelli S, Bai G, Li R, Von Korff M, Varshney RK, Graner A, Valkoun J: Differentially expressed genes between droughttolerant and drought-sensitive barley genotypes in response to drought stress during the reproductive stage. J Exp Biol 2009, 60:3531-3544.

59. Sun J, Jiang H, Xu Y, Li H, Wu X, Xie Q, Li C: The CCCH-type zinc finger proteins AtSZF1 and AtSZF2 regulate salt stress responses in Arabidopsis. Plant Cell Physiol 2007, 48:1148-1158.

60. Zhou QY, Tian AG, Zou HF, Xie ZM, Lei G, Huang J, Wang CM, Wang HW, Zhang JS, Chen SY: Soybean WRKY-type transcription factor genes, GmWRKY13, GmWRKY21, and GmWRKY54, confer differential tolerance to abiotic stresses in transgenic Arabidopsis plants. Plant Biotechnol J 2008, 6:486-503.

61. Zou M, Guan Y, Ren H, Zhang F, Chen F: A bZIP transcription factor, OsABI5, is involved in rice fertility and stress tolerance. Plant Mol Biol 2008, 66:675-683.

62. Meng X, Li F, Liu C, Zhang C, Wu Z, Chen Y: Isolation and characterization of an ERF transcription factor gene from cotton (Gossypium barbadense L.). Plant Mol Biol Rep 2010, 28:176-183.

63. Michalak P: Coexpression, coregulation, and cofunctionality of neighboring genes in eukaryotic genomes. Genomics 2008, 91:243-248.

64. Ge Y, Li Y, Lv D-K, Bai X, Ji W, Cai H, Wang A-X, Zhu Y-M: Alkaline-stress response in Glycine soja leaf identifies specific transcription factors and ABA-mediated signaling factors. Funct Integr Genomic 2011, 11:369-379.

65. Büttner M, Singh KB: Arabidopsis thaliana ethylene-responsive element binding protein (AtEBP), an ethylene-inducible, GCC box DNA-binding protein interacts with an ocs element binding protein. Proc Natl Acad Sci US A 1997, 94:5961-5966.

66. Licausi F, Kosmacz M, Weits DA, Giuntoli B, Giorgi FM, Voesenek LA, Perata $P$, van Dongen JT: Oxygen sensing in plants is mediated by an $\mathrm{N}$-end rule pathway for protein destabilization. Nature 2011, 479:419-422. 
67. Meng C-M, Zhang T-Z, Guo W-Z: Molecular cloning and characterization of a novel Gossypium hirsutum L. bHLH gene in response to ABA and drought stresses. Plant Mol Biol Rep 2009, 27:381-387.

68. Qin Y-F, Li D-D, Wu Y-J, Liu Z-H, Zhang J, Zheng Y, Li X-B: Three cotton homeobox genes are preferentially expressed during early seedling development and in response to phytohormone signaling. Plant Cell Rep 2010, 29:1147-1156.

69. Ni Y, Wang X, Li D, Wu Y, Xu W, Li X: Novel cotton homeobox gene and its expression profiling in root development and in response to stresses and phytohormones. Acta Bioch Bioph Sin 2008, 40:78-84.

70. Sakamoto H, Maruyama K, Sakuma Y, Meshi T, Iwabuchi M, Shinozaki K, Yamaguchi-Shinozaki K: Arabidopsis Cys2/His2-type zinc-finger proteins function as transcription repressors under drought, cold, and high-salinity stress conditions. Plant Physiol 2004, 136:2734-2746.

71. Huang J, Sun S-J, Xu D-Q, Yang X, Bao Y-M, Wang Z-F, Tang H-J, Zhang H: Increased tolerance of rice to cold, drought and oxidative stresses mediated by the overexpression of a gene that encodes the zinc finger protein ZFP245. Biochem Bioph Res Co 2009, 389:556-561.

72. Lightfoot DJ, Malone KM, Timmis JN, Orford SJ: Evidence for alternative splicing of MADS-box transcripts in developing cotton fibre cells. Molecul Genet Genomics 2008, 279:75-85.

73. Sakuma Y, Maruyama K, Qin F, Osakabe Y, Shinozaki K, Yamaguchi-Shinozaki $K$ : Dual function of an Arabidopsis transcription factor DREB2A in water-stress-responsive and heat-stress-responsive gene expression. ProC Natl Acad Sci U S A 2006, 103:18822-18827.

74. Sakuma Y, Maruyama K, Osakabe Y, Qin F, Seki M, Shinozaki K, Yamaguchi-Shinozaki K: Functional analysis of an Arabidopsis transcription factor, DREB2A, involved in drought-responsive gene expression. Plant Cell 2006, 18:1292-1309.

75. Nakashima K, Shinwari ZK, Sakuma Y, Seki M, Miura S, Shinozaki K, Yamaguchi-Shinozaki K: Organization and expression of two Arabidopsis DREB2 genes encoding DRE-binding proteins involved in dehydration-and high-salinity-responsive gene expression. Plant Mol Biol 2000, 42:657-665.

76. Shan DP, Huang JG, Yang YT, Guo YH, Wu CA, Yang GD, Gao Z, Zheng CC: Cotton GhDREB1 increases plant tolerance to low temperature and is negatively regulated by gibberellic acid. New Phytol 2007, 176:70-81.

77. Servet C, Benhamed M, Latrasse D, Kim W, Delarue M, Zhou D-X: Characterization of a phosphatase $2 \mathrm{C}$ protein as an interacting partner of the histone acetyltransferase GCN5 in Arabidopsis. Biochim Biophys Acta 2008, 1779:376-382.

78. Jamil M, labal W, Bangash A, Rehman SU, Imran QM, Rha ES: Constitutive expression of $\mathrm{OSC} 3 \mathrm{H} 33, \mathrm{OSC} 3 \mathrm{H} 50$ and $\mathrm{OSC} 3 \mathrm{H} 37$ genes in rice under salt stress. Pak J Bot 2010, 42:4003-4009.

79. Gobert A, Park G, Amtmann A, Sanders D, Maathuis FJ: Arabidopsis thaliana cyclic nucleotide gated channel 3 forms a non-selective ion transporter involved in germination and cation transport. J Exp Biol 2006, 57:791-800

80. Mahajan S, Pandey GK, Tuteja N: Calcium-and salt-stress signaling in plants: shedding light on SOS pathway. Arch Biochem Biophys 2008, 471:146-158.

81. Xiong L, Schumaker KS, Zhu J-K: Cell signaling during cold, drought, and salt stress. Plant Cell 2002, 14(suppl 1):S165-S183.

82. Weinl S, Kudla J: The CBL-CIPK Ca ${ }^{2+}$-decoding signaling network: function and perspectives. New Phytol 2009, 184:517-528.

83. H-i C, Park H-J, Park JH, Kim S, Im M-Y, Seo H-H, Kim Y-W, Hwang I, Kim SY: Arabidopsis calcium-dependent protein kinase AtCPK32 interacts with ABF4, a transcriptional regulator of abscisic acid-responsive gene expression, and modulates its activity. Plant Physiol 2005, 139:1750-1761.

84. Zhen J, Zhang X, Wang Y, Zhai Z, Li Z, Hua J: Isolation and sequence analysis of salt responsive gene GhCPK5 from Gossypium hirsutum L. J Chin Agric Univ 2011, 16:8-14.

85. Saijo Y, Hata S, Kyozuka J, Shimamoto K, Izui K: Over expression of a single Ca2+-dependent protein kinase confers both cold and salt/drought tolerance on rice plants. Plant J 2000, 23:319-327.

86. Sheen $\mathrm{J}: \mathrm{Ca}^{2+}$-dependent protein kinases and stress signal transduction in plants. Science 1996, 274:1900-1902.

87. Teige M, Scheikl E, Eulgem T, Dóczi R, Ichimura K, Shinozaki K, Dangl JL, Hirt $\mathrm{H}$ : The MKK2 Pathway Mediates Cold and Salt Stress Signaling in Arabidopsis. Mol Cell 2004, 15:141-152.

88. Luo J, Zhao L-L, Gong S-Y, Sun X, Li P, Qin L-X, Zhou Y, Xu W-L, Li X-B: A cotton mitogen-activated protein kinase (GhMPK6) is involved in ABA-induced CAT1 expression and $\mathrm{H}_{2} \mathrm{O}_{2}$ production. J Genet Genomics 2011, 38:557-565.
89. Shi J, An H-L, Zhang L, Gao Z, Guo X-Q: GhMPK7, a novel multiple stress-responsive cotton group C MAPK gene, has a role in broad spectrum disease resistance and plant development. Plant Mol Biol 2010, 74:1-17.

90. Peleg Z, Blumwald E: Hormone balance and abiotic stress tolerance in crop plants. Curr Opin Plant Biol 2011, 14:290-295.

91. Seo M, Koshiba T: Complex regulation of ABA biosynthesis in plants. Trends Plant Sci 2002, 7:41-48.

92. Chen C-N, Chu C-C, Zentella R, Pan S-M, Ho T-HD: AtHVA22 gene family in Arabidopsis: phylogenetic relationship, $\mathrm{ABA}$ and stress regulation, and tissue-specific expression. Plant Mol Biol 2002, 49:631-642.

93. Koops P, Pelser S, Ignatz M, Klose C, Marrocco-Selden K, Kretsch T: EDL3 is an $\mathrm{F}$-box protein involved in the regulation of abscisic acid signalling in Arabidopsis thaliana. J Exp Biol 2011, 62:5547-5560.

94. Hirayama T, Shinozaki K: Perception and transduction of abscisic acid signals: keys to the function of the versatile plant hormone ABA. Trends Plant Sci 2007, 12:343-351.

95. Yoo S-D, Cho Y-H, Tena G, Xiong Y, Sheen J: Dual control of nuclear EIN3 by bifurcate MAPK cascades in $\mathrm{C} 2 \mathrm{H} 4$ signalling. Nature 2008, 451:789-795.

96. Padmalatha KV, Dhandapani G, Kanakachari M, Kumar S, Dass A, Patil DP, Rajamani V, Kumar K, Pathak R, Rawat B: Genome-wide transcriptomic analysis of cotton under drought stress reveal significant downregulation of genes and pathways involved in fibre elongation and up-regulation of defense responsive genes. Plant Mol Biol 2012, 78:223-246.

97. Vanlerberghe GC, Cvetkovska M, Wang J: Is the maintenance of homeostatic mitochondrial signaling during stress a physiological role for alternative oxidase? Physiol Plantarum 2009, 137:392-406.

98. Kirch H-H, Schlingensiepen S, Kotchoni S, Sunkar R, Bartels D: Detailed expression analysis of selected genes of the aldehyde dehydrogenase (ALDH) gene superfamily in Arabidopsis thaliana. Plant Mol Biol 2005, 57:315-332.

99. Kotchoni SO, Kuhns C, Ditzer A, KIRCH HH, Bartels D: Over expression of different aldehyde dehydrogenase genes in Arabidopsis thaliana confers tolerance to abiotic stress and protects plants against lipid peroxidation and oxidative stress. Plant Cell Environ 2006, 29:1033-1048.

100. Sunkar R: MicroRNAs with macro-effects on plant stress responses. In Seminars in cell \& developmental biology: Academic Press; 2010. 21: 805-811.

101. Li B, Qin Y, Duan H, Yin W, Xia X: Genome-wide characterization of new and drought stress responsive microRNAs in Populus euphratica. J Exp Biol 2011, 62:3765-3779.

102. Zhang Q, Zhao C, Li M, Sun W, Liu Y, Xia H, Sun M, Li A, Li C, Zhao S: Genome-wide identification of Thellungiella salsuginea microRNAs with putative roles in the salt stress response. BMC Plant Biol 2013, 13:180.

103. Glazov EA, Cottee PA, Barris WC, Moore RJ, Dalrymple BP, Tizard ML: A microRNA catalog of the developing chicken embryo identified by a deep sequencing approach. Genome Res 2008, 18:957-964.

104. Sunkar R, Chinnusamy V, Zhu J, Zhu J-K: Small RNAs as big players in plant abiotic stress responses and nutrient deprivation. Trends Plant Sci 2007, 12:301-309.

105. Lelandais-Brière C, Sorin C, Declerck M, Benslimane A, Crespi M, Hartmann C: Small RNA diversity in plants and its impact in development. Curr Genomics 2010, 11:14-23.

106. Wu G, Park MY, Conway SR, Wang J-W, Weigel D, Poethig RS: The Sequential Action of miR156 and miR172 Regulates Developmental Timing in Arabidopsis. Cell 2009, 138:750-759.

107. Olsen AN, Ernst HA, Leggio LL, Skriver K: NAC transcription factors: structurally distinct, functionally diverse. Trends Plant Sci 2005, 10:79-87.

108. Xie Q, Frugis G, Colgan D, Chua N-H: Arabidopsis NAC1 transduces auxin signal downstream of TIR1 to promote lateral root development. Gene Dev 2000, 14:3024-3036.

109. Duval M, Hsieh T-F, Kim SY, Thomas TL: Molecular characterization of AtNAM: A member of the Arabidopsis NAC domain superfamily. Plant Mol Biol 2002, 50:237-248.

110. Nikovics K, Blein T, Peaucelle A, Ishida T, Morin H, Aida M, Laufs P: The balance between the MIR164A and CUC2 genes controls leaf margin serration in Arabidopsis. Plant Cell 2006, 18:2929-2945.

111. Meng Y, Ma X, Chen D, Wu P, Chen M: MicroRNA-mediated signaling involved in plant root development. Biochem Bioph Res Co 2010, 393:345-349. 
112. Toledo-Ortiz G, Huq E, Quail PH: The Arabidopsis basic/helix-loop-helix transcription factor family. Plant Cell 2003, 15:1749-1770.

113. X-M DU, J-L SUN, Z-L ZHOU, Y-H GU, Z-E PAN, S-P HE, B-Y PANG, L-R WANG Current situation and the future in collection, preservation, evaluation and utilization of cotton germplasm in China. J Plant Genet Resour 2012, 13(2):163-168

114. Hoagland DR, Arnon DI: The water-culture method for growing plants without soil. Calif Agric Exp Stn Circ 1950, 347(2nd edit).

115. Smart RE, Bingham GE: Rapid estimates of relative water content. Plant Physiol 1974, 53:258-260.

116. Cao W-H, Liu J, He X-J, Mu R-L, Zhou H-L, Chen S-Y, Zhang J-S: Modulation of ethylene responses affects plant salt-stress responses. Plant Physiol 2007, 143:707-719.

117. Porra R, Thompson W, Kriedemann P: Determination of accurate extinction coefficients and simultaneous equations for assaying chlorophylls $a$ and $b$ extracted with four different solvents: verification of the concentration of chlorophyll standards by atomic absorption spectroscopy. BBA-Bioenergetics 1989, 975:384-394.

118. Livak KJ, Schmittgen TD: Analysis of Relative Gene Expression Data Using Real-Time Quantitative PCR and the 2- $\Delta \Delta C T$ Method. Methods 2001, 25:402-408

119. Kosmas SA, Argyrokastritis A, Loukas MG, Eliopoulos E, Tsakas S, Kaltsikes PJ: Isolation and characterization of drought-related trehalose 6-phosphate-synthase gene from cultivated cotton (Gossypium hirsutum L). Planta 2006, 223:329-339.

120. Chen C, Ridzon DA, Broomer AJ, Zhou Z, Lee DH, Nguyen JT, Barbisin M, Xu NL, Mahuvakar VR, Andersen MR: Real-time quantification of microRNAs by stem-loop RT-PCR. Nucleic Acids Res 2005, 33:e179-e179.

121. Varkonyi-Gasic E, Wu R, Wood M, Walton EF, Hellens RP: Protocol: a highly sensitive RT-PCR method for detection and quantification of microRNAs. Plant Methods 2007, 3:12.

122. Grabherr MG, Haas BJ, Yassour M, Levin JZ, Thompson DA, Amit I, Adiconis X, Fan L, Raychowdhury R, Zeng Q: Full-length transcriptome assembly from RNA-Seq data without a reference genome. Nat Biotechnol 2011, 29:644-652.

123. Conesa A, Götz S, García-Gómez JM, Terol J, Talón M, Robles M: Blast2GO: a universal tool for annotation, visualization and analysis in functional genomics research. Bioinformatics 2005, 21:3674-3676.

124. Ye J, Fang L, Zheng H, Zhang Y, Chen J, Zhang Z, Wang J, Li S, Li R, Bolund L: WEGO: a web tool for plotting GO annotations. Nucleic Acids Res 2006 34(suppl 2):W293-W297.

125. Mortazavi A, Williams BA, McCue K, Schaeffer L, Wold B: Mapping and quantifying mammalian transcriptomes by RNA-Seq. Nat Methods 2008, 5:621-628.

126. Audic S, Claverie J-M: The significance of digital gene expression profiles. Genome Res 1997, 7:986-995.

127. Ernst J, Bar-Joseph Z: STEM: a tool for the analysis of short time series gene expression data. BMC Bioinformatics 2006, 7:191.

128. Man MZ, Wang $X$, Wang Y: POWER_SAGE: comparing statistical tests for SAGE experiments. Bioinformatics 2000, 16:953-959.

doi:10.1186/1471-2164-15-760

Cite this article as: Peng et al:: Comprehensive analysis of differentially expressed genes and transcriptional regulation induced by salt stress in two contrasting cotton genotypes. BMC Genomics 2014 15:760.

\section{Submit your next manuscript to BioMed Central and take full advantage of:}

- Convenient online submission

- Thorough peer review

- No space constraints or color figure charges

- Immediate publication on acceptance

- Inclusion in PubMed, CAS, Scopus and Google Scholar

- Research which is freely available for redistribution

Submit your manuscript at www.biomedcentral.com/submit
Ciomed Central 